\title{
Symmetric interpolatory dual wavelet frames *
}

\author{
A.V. Krivoshein \\ Department of Applied Mathematics and Control Processes, \\ St. Petersburg State University \\ KrivosheinAV@gmail.com, a.krivoshein@spbu.ru
}

\begin{abstract}
For any symmetry group $H$ and any appropriate matrix dilation we give an explicit method for the construction of $H$-symmetric refinable interpolatory refinable masks which satisfy sum rule of arbitrary order $n$. For each such mask we give an explicit technique for the construction of dual wavelet frames such that the corresponding wavelet masks are mutually symmetric and have the vanishing moments up to the order n. For an abelian symmetry group $H$ we modify the technique such that each constructed wavelet mask is $H$-symmetric.
\end{abstract}

Keywords dual wavelet frames, Mixed Extension Principle, interpolatory mask, symmetry group AMS Subject Classification: $42 \mathrm{C} 40$

\section{Introduction}

The symmetry of refinable and wavelet functions is one of the important feature in applications. A lot of papers are devoted to the construction of wavelets with the symmetry properties. In the univariate case the general methods providing the symmetry for wavelets were presented in [12, 23, (in [15, 28] for multiwavelets) and the references therein. But in the multivariate case to the best knowledge of the author there are no general methods for the construction of wavelet systems with an arbitrary symmetry properties and for any appropriate dilation matrices. The problem in this case is complicated by the fact that there are different kinds of symmetry. Also, unlike the univariate case, in the multivariate case there are no simple and explicit general algorithms for deriving the wavelet systems from the refinable functions. The variety of methods for the construction of wavelets in the different setups in the multivariate case can be found in [5, [13, 21, etc. (and the reference therein).

It seems impossible to provide a complete list of references about the construction of symmetric wavelets. The following synopsis just briefly highlight some investigations on the problem. The symmetry properties of functions can be defined using the notion of symmetry group (for details see Section 2.1 and, also, [11). Let $H$ be a symmetry group. In [1] G. Andaloro and co-authors suggest a method for the construction of $H$-symmetric wavelet systems starting with refinable interpolatory masks. The method is based on the lifting scheme; the choice of dilation matrix $M$ is restricted only to $M=2 I_{d}$. K. Koch in [19] give an algorithm for the construction of interpolating scaling vectors with the $H$-symmetry property. The multiwavelets are also constructed using certain technique that does not care about the symmetry properties. In several examples it turns out that the multiwavelets are mutually symmetric. In this paper we clarify this effect in the case of scalar wavelets. Some schemes for the construction of highly symmetric wavelet systems were presented by Q. T. Jiang and

\footnotetext{
${ }^{*}$ This research was supported by the RFBR-grant (\#12-01-00216-a) and by the grant of the St.Petersburg State University (\#9.38.62.2012).
} 
D.K. Pounds in 17]. In [18, M. Skopina and co-authors suggest a method for the construction of refinable interpolatory masks and wavelet masks which are point-symmetric (point-antisymmetric) for several dilation matrices. In 20] the point and axial symmetric frame-like wavelet systems were constructed. The aim of this paper is to extend the results in [18, and to generalize the method in [20] on any symmetry group and any appropriate dilation matrix in order to construct the $H$-symmetric dual wavelet frames generated by interpolatory refinable masks.

Besides the symmetry property, there are another important features for applications such as the good approximation properties of wavelet system, the smoothness and the small support of refinable and wavelet functions. The good approximation property of wavelet system is connected with the orders of vanishing moments of the wavelet functions, which in turn are connected with the orders of sum rule of the refinable masks. The desirable order of vanishing moments for the wavelet functions will be explicitly incorporated in the proposed construction. The order of smoothness of the refinable functions does not incorporated in the construction. But the Sobolev smoothness exponent can be computed ex post, using the efficient algorithm developed by B. Han in [10]. The analysis of smoothness and its dependence from the mask support can be found in [6]. Some facts about how the symmetry affects on the smoothness can be found in [7].

The paper is organized as follows. In Section 2 we give some notations and the notion of symmetry group. In Section 3 we recall the Mixed Extension Principle. The main results are contained in Section 4. Subsection 4.1 is devoted to the construction of interpolatory $H$-symmetric refinable masks. Subsection 4.2 describes the construction of mutually symmetric wavelets. In Subsection 4.3 we present a method for the construction of wavelet masks such that all of them have the $H$-symmetry properties. In Section 5 several examples are presented.

\section{Notation}

$\mathbb{N}$ is the set of positive integers, $\mathbb{R}$ is the set of real numbers, $\mathbb{C}$ is the set of complex numbers. $\mathbb{R}^{d}$ is the $d$-dimensional Euclidean space, $x=\left(x_{1}, \ldots, x_{d}\right)$ and $y=\left(y_{1}, \ldots, y_{d}\right)$ are its elements (vectors), $(x)_{j}=x_{j}$ for $j=1, \ldots, d,(x, y)=x_{1} y_{1}+\ldots+x_{d} y_{d},|x|=\sqrt{(x, x)}, \mathbf{0}=(0, \ldots, 0) \in \mathbb{R}^{d}$. For $x, y \in \mathbb{R}^{d}$ we write $x>y$ if $x_{j}>y_{j}, j=1, \ldots, d ; x \geq y$ if $x_{j} \geq y_{j}, j=1, \ldots, d$. $\mathbb{Z}^{d}$ is the integer lattice in $\mathbb{R}^{d}, \mathbb{Z}_{+}^{d}:=\left\{x \in \mathbb{Z}^{d}: x \geq \mathbf{0}\right\}$. If $\alpha, \beta \in \mathbb{Z}_{+}^{d}, a, b \in \mathbb{R}^{d}$, we set $[\alpha]=\sum_{j=1}^{d} \alpha_{j}, \alpha !=\prod_{j=1}^{d} \alpha_{j}$ !, $\left(\begin{array}{l}\beta \\ \alpha\end{array}\right)=\frac{\alpha !}{\beta !(\alpha-\beta) !}, a^{b}=\prod_{j=1}^{d} a_{j}^{b_{j}}, D^{\alpha} f=\frac{\partial^{[\alpha]} f}{\partial x^{\alpha}}=\frac{\partial^{[\alpha]} f}{\partial^{\alpha} x_{1} \ldots \partial^{\alpha} d x_{d}}, \delta_{a b}$ is the Kronecker delta. Suppose $n \in \mathbb{N}$, then $\Delta_{n}:=\left\{\alpha: \alpha \in \mathbb{Z}_{+}^{d},[\alpha]<n\right\}$. Suppose $\beta \in \mathbb{Z}_{+}^{d}$, then $\square_{\beta}:=\left\{\alpha: \alpha \in \mathbb{Z}_{+}^{d}, \alpha \leq \beta\right\}$. By \#H denote the cardinality of the set $H$. By $\operatorname{diag}\left(u_{1}, \ldots, u_{d}\right), u_{1}, \ldots, u_{d} \in \mathbb{Z}$, denote the $d \times d$ diagonal matrix such that numbers $u_{1}, \ldots, u_{d}$ are placed on the main diagonal. The $(\mathrm{i}, \mathrm{j})$-th element of matrix $M$ is denoted by $[M]_{i, j}$

An integer $d \times d$ matrix $M$ is called a dilation matrix if the eigenvalues of $M$ are bigger than 1 in module. By $M^{*}$ denote the transpose conjugate matrix $M$, by $I_{d}$ denote the $d \times d$ identity matrix, $m=|\operatorname{det} M|$. We say that the vectors $\alpha, \beta \in \mathbb{Z}^{d}$ are congruent modulo $M$ if $\alpha-\beta=M \gamma, \gamma \in \mathbb{Z}^{d}$. The integer lattice $\mathbb{Z}^{d}$ is split into cosets with respect to the congruence. It is known that the number of the cosets is equal to $m$ (see, e.g., [22, § 2.2]). Let us choose an arbitrary representative from each coset, call them all digits, and denote by $D(M)=\left\{s_{0}, \ldots, s_{m-1}\right\}$ the set of digits. Thus, any $\alpha \in \mathbb{Z}^{d}$ can be uniquely represented as $\alpha=M \beta+s_{k}$, where $\beta \in \mathbb{Z}^{d}, s_{k} \in D(M)$. Throughout the paper we assume that $s_{0}=\mathbf{0}$. Denote by $\langle s\rangle$ the coset containing digit $s \in D(M)$ or, equivalently, $\langle s\rangle:=\left\{M \beta+s, \beta \in \mathbb{Z}^{d}\right\}$.

Let $f$ be a function defined on $\mathbb{R}^{d}$; then $f_{j k}=m^{j / 2} f\left(M^{j} \cdot+k\right)$, where $j \in \mathbb{Z}, k \in \mathbb{Z}^{d}$, and $\left\{f_{j k}\right\}:=\left\{f_{j k}: j \in \mathbb{Z}, k \in \mathbb{Z}^{d}\right\}$. The Fourier transform of $f \in L_{1}\left(\mathbb{R}^{d}\right)$ is defined to be $\widehat{f}(\xi)=$ $\int_{\mathbb{R}^{d}} f(x) e^{-2 \pi i(x, \xi)} d x, \xi \in \mathbb{R}^{d}$. This notion can be naturally extended to $L_{2}\left(\mathbb{R}^{d}\right)$. 
Let $S^{\prime}$ denote the space of tempered distributions. For any $f \in S^{\prime}$, its Sobolev smoothness exponent is defined to be

$$
\nu_{2}(f)=\sup \left\{\nu \in \mathbb{R} \bigcup\{-\infty\} \bigcup\{+\infty\}: \int_{\mathbb{R}^{d}}|\widehat{f}(\xi)|^{2}\left(1+|\xi|^{2}\right)^{\nu} d \xi<\infty\right\} .
$$

A trigonometric polynomial $t(\xi), \xi \in \mathbb{R}^{d}$, is a finite linear combination of complex exponentials, i.e. $t(\xi)=\sum_{k \in \mathbb{Z}^{d}} h_{k} e^{2 \pi i(k, \xi)}, h_{k} \in \mathbb{C}$. A shifted trigonometric polynomial $t^{\prime}(\xi), \xi \in \mathbb{R}^{d}$, is defined by $t^{\prime}(\xi)=e^{2 \pi i(\sigma, \xi)} t(\xi) \sigma \in \mathbb{R}$.

A function/distribution $\varphi$ is called refinable if there exists a 1-periodic function $m_{0} \in L_{2}\left([0,1]^{d}\right)$ (refinable mask, also low-pass filter) such that

$$
\widehat{\varphi}(\xi)=m_{0}\left(M^{*-1} \xi\right) \widehat{\varphi}\left(M^{*-1} \xi\right) .
$$

This condition is called the refinement equation. It is well known (see, e.g., [22, § 2.4]) that for any trigonometric polynomial $m_{0}$ satisfying $m_{0}(\mathbf{0})=1$ there exists a unique (up to a factor) solution of the refinement equation (1) in $S^{\prime}$. The solution is compactly supported and it is given by its Fourier transform

$$
\widehat{\varphi}(\xi):=\prod_{j=1}^{\infty} m_{0}\left(M^{*-j} \xi\right) .
$$

Throughout the paper we assume that any refinable mask $m_{0}$ is a trigonometric polynomial with real or complex coefficients and $m_{0}(\mathbf{0})=1$.

Let us fix a dilation matrix $M$ and its digits. For any trigonometric polynomial $t$ there exists a unique set of trigonometric polynomials $\tau_{k}, k=0, \ldots, m-1$, such that

$$
t(\xi)=\frac{1}{\sqrt{m}} \sum_{k=0}^{m-1} e^{2 \pi i\left(s_{k}, \xi\right)} \tau_{k}\left(M^{*} \xi\right),
$$

where $s_{k}$ are the digits of $M$. Equality (3) is the polyphase representation of $t$. For each $k=$ $0, \ldots, m-1$ the trigonometric polynomial $\tau_{k}$ is called the polyphase component of $t$ corresponding to the digit $s_{k}$. Changing the set of digits will also change the polyphase components of the trigonometric polynomial $t$ (namely, they will be shifted by certain integer shifts). Let us remark that the polyphase components can be explicitly defined by (see, e.g., [22, § 2.6])

$$
\tau_{k}(\xi)=\frac{1}{\sqrt{m}} \sum_{s \in D\left(M^{*}\right)} e^{-2 \pi i\left(M^{-1} s_{k}, \xi+s\right)} t\left(M^{*-1}(\xi+s)\right), \quad k=0, \ldots, m-1 .
$$

Let $t$ be a trigonometric polynomial. We say that $t$ is interpolatory if

$$
\sum_{s \in D\left(M^{*}\right)} t\left(\xi+M^{*-1} s\right) \equiv 1 .
$$

It follows from (4) that for the interpolatory trigonometric polynomial $t$ its polyphase component corresponding to the digit $s_{0}=\mathbf{0}$ is a constant, namely $\tau_{0} \equiv \frac{1}{\sqrt{m}}$.

Let $n \in \mathbb{N}$. We say that a trigonometric polynomial $t$ has sum rule of order $n$ with respect to the dilation matrix $M$ if

$$
\left.D^{\beta} t\left(M^{*-1} \xi\right)\right|_{\xi=s}=0, \quad \forall s \in D\left(M^{*}\right) \backslash\{\mathbf{0}\}, \quad \forall \beta \in \Delta_{n} .
$$

The order of sum rule is a very important feature in applications, since it is connected with the good approximation properties of the corresponding wavelet systems (see, e.g. [16] and the references therein). Condition (5) can be formulated via the polyphase components of trigonometric polynomial $t$. 
Lemma 1 (4. Theorem 11]) A trigonometric polynomial $t$ has sum rule of order $n \in \mathbb{N}$ if and only if its polyphase components $\tau_{k}, k=0, \ldots, m-1$, satisfy

$$
D^{\beta} \tau_{k}(\mathbf{0})=\frac{(2 \pi i)^{[\beta]}}{\sqrt{m}} \sum_{\alpha \in \square_{\beta}} \lambda_{\alpha}\left(\begin{array}{c}
\beta \\
\alpha
\end{array}\right)\left(-M^{-1} s_{k}\right)^{\beta-\alpha} \quad \forall \beta \in \Delta_{n},
$$

where $\lambda_{\alpha} \in \mathbb{C}$ are defined by

$$
\lambda_{\alpha}=\left.\frac{1}{(2 \pi i)^{[\alpha]}} D^{\alpha} t\left(M^{*-1} \xi\right)\right|_{\xi=\mathbf{0}}, \quad \alpha \in \Delta_{n} .
$$

Note that if trigonometric polynomial $t$ has sum rule of order 1 and $t(\mathbf{0})=1$ then condition (6) is just $\tau_{k}(\mathbf{0})=\frac{1}{\sqrt{m}}, k=0, \ldots, m-1, \lambda_{\mathbf{0}}=1$.

For an interpolatory trigonometric polynomial $t$ Lemma 1 can be simplified. Since $\tau_{0}=\frac{1}{\sqrt{m}}$, then from condition (6) for $\tau_{0}$ we get that $\lambda_{\alpha}=\delta_{\mathbf{0} \alpha}, \alpha \in \Delta_{n}$. Thus, the conditions on the polyphase components of $t$ are

$$
D^{\beta} \tau_{k}(\mathbf{0})=\frac{(2 \pi i)^{[\beta]}}{\sqrt{m}}\left(-M^{-1} s_{k}\right)^{\beta} \quad \forall \beta \in \Delta_{n}
$$

where $k=0, \ldots, m-1$. Also by (7) we have that $\left.D^{\alpha} t\left(M^{*-1} \xi\right)\right|_{\xi=\mathbf{0}}=\delta_{\mathbf{0} \alpha}, \alpha \in \Delta_{n}$.

We say that a trigonometric polynomial $t$ has vanishing moments of order $n$ if

$$
\left.D^{\beta} t(\xi)\right|_{\xi=\mathbf{0}}=0, \quad \forall \beta \in \Delta_{n} .
$$

Finally, we say that $t$ has linear-phase moments of order $n$ with phase $c \in \mathbb{R}^{d}$ if

$$
D^{\beta} t(\mathbf{0})=\left.D^{\beta} e^{2 \pi i(c, \xi)}\right|_{\xi=\mathbf{0}}=(2 \pi i)^{[\beta]} c^{\beta}, \quad \forall \beta \in \Delta_{n} .
$$

This notion was described in [14] for the univariate case and in [16] for more general settings. The importance of the linear-phase moments is in the fact that they are useful in the setting of polynomial reproduction and subdivision schemes.

Notice that the above properties of trigonometric polynomials are invariant with respect to the integer shifts. If a trigonometric polynomial $t$ has sum rule of order $n$ (or vanishing moments of order $n$, or linear-phase moments of order $n$ with phase $\left.c \in \mathbb{R}^{d}\right)$ then $t^{\prime}(\xi):=t(\xi) e^{2 \pi i(\gamma, \xi)}, \gamma \in \mathbb{Z}^{d}$, also has sum rule of order $n$ (or vanishing moments of order $n$, or linear-phase moments of order $n$ but with phase $c+\gamma)$.

For functions $\psi^{(\nu)}, \nu=1, \ldots, r$, the system of their dilations and translations $\left\{\psi_{j k}^{(\nu)}\right\}$ is called a wavelet system. Suppose $n \in \mathbb{N}$. We say that the wavelet system $\left\{\psi_{j k}^{(\nu)}\right\}$ has vanishing moments of order $n$ (or has a $V M^{n}$ property) if

$$
D^{\beta} \widehat{\psi^{(\nu)}}(\mathbf{0})=0, \quad \nu=1, \ldots, r, \quad \forall \beta \in \Delta_{n} .
$$

A family of functions $\left\{f_{\alpha}\right\}_{\alpha \in \aleph}$ ( $\aleph$ is a countable index set) in a Hilbert space $H$ is called a frame in $H$ if there exist constants $A, B>0$ such that

$$
A\|f\|^{2} \leq \sum_{\alpha \in \aleph}\left|\left\langle f, f_{\alpha}\right\rangle\right|^{2} \leq B\|f\|^{2}, \quad \forall f \in H .
$$

The important property of any frame $\left\{f_{\alpha}\right\}_{\alpha}$ in $H$ is the following: every $f \in H$ can be decomposed as $f=\sum_{\alpha}\left\langle f, \widetilde{f}_{\alpha}\right\rangle f_{\alpha}$, where $\left\{\widetilde{f}_{\alpha}\right\}_{\alpha}$ is a dual frame in $H$. The comprehensive characterization of 
frames can be found in [3]. The wavelet frames are of great interest in many applications, especially in signal processing.

Further, we give some results from the theory of permutation groups (see, e.g., [2]). Let $H$ be a finite group (with a binary operation ".") and let $\Omega$ be a finite set. A group action of $H$ on $\Omega$ is a map $\chi: H \times \Omega \longrightarrow \Omega$ such that the following conditions hold for any element $\omega \in \Omega$ :

1. the identity, i.e. $\chi(I, \omega)=\omega$, where $I$ is the identity element of $H$;

2. the associativity, i.e. $\chi(E, \chi(\widetilde{E}, \omega))=\chi(E \cdot \widetilde{E}, \omega)$ for all $E, \widetilde{E} \in H$.

The set $\Omega$ is called an $H$-space if there exist a group action $\chi$ of $H$ on $\Omega$. A group action of $H$ on $\Omega$ somehow permutes the elements of $\Omega$.

Suppose $\Omega$ is an $H$-space and $\omega \in \Omega$. Denote the map $\chi(E, \omega)$ by $E \omega$. The set $H \omega:=\{E \omega, E \in$ $H\}$ is called the orbit of $\omega$. Not hard to see that two orbits are either equal or disjoint. Thus, $\Omega$ can be represented as the union of disjoint orbits.

Lemma 2 Suppose $\Omega$ is an $H$-space. Then there exists a set $\Lambda \subset \Omega$ such that $\Omega=\cup_{\omega \in \Lambda} H \omega$ and the orbits $H \omega, \omega \in \Lambda$, are mutually disjoint.

The subgroup $H_{\omega}$ of $H$ defined by $H_{\omega}:=\{E \in H: E \omega=\omega\}$ is called the stabilizer of $\omega . H \backslash H_{\omega}$ is the quotient group of $H$ modulo $H_{\omega}$. The orbit of $\omega$ is isomorphic to $H \backslash H_{\omega}$. Denote by $\Gamma_{\omega}$ a complete set of representatives of the cosets of $H \backslash H_{\omega}$. Then, the orbit of $\omega$ can be represented as $H \omega=\cup_{E \in \Gamma_{\omega}} E \omega$. Hence, the set $\Omega$ can be decomposed as

$$
\Omega=\cup_{\omega \in \Lambda} \cup_{E \in \Gamma_{\omega}} E \omega,
$$

where the set $\Lambda$ is from Lemma 2. Also note that $\# \Gamma_{\omega}=\# H \omega$, the set $\Gamma_{\omega}$ and $H_{\omega}$ consists of the elements of $H$, the set $H \omega$ consists of the elements of $\Omega$.

Lemma 3 Suppose $\Omega$ is an $H$-space, $\omega \in \Omega$. Let $\Gamma_{\omega}$ be a complete set of representatives of the cosets of $H \backslash H_{\omega}$. Then $\# H=\# H_{\omega} \cdot \# \Gamma_{\omega}$ and each $\widetilde{E} \in H$ can be uniquely represented as $\widetilde{E}=E \cdot F$, where $E \in \Gamma_{\omega}, F \in H_{\omega}$.

Suppose $\omega \in \Omega, \eta \in H \omega$, i.e. there exist $E \in H$ such that $\eta=E \omega$. Then the stabilizer of $\eta$ and the stabilizer of $\omega$ are conjugate subgroups, namely $E H_{\omega} E^{-1}=H_{\eta}$.

\subsection{Symmetry property of masks and refinable functions}

A finite set $H$ of $d \times d$ unimodular matrices (i.e. square integer matrices with determinant \pm 1 ) is a symmetry group on $\mathbb{Z}^{d}$ if $H$ forms a group under the matrix multiplication. Let $H$ be a symmetry group on $\mathbb{Z}^{d}$. A function $f$ is called $H$-symmetric with respect to the center $C \in \mathbb{R}^{d}$ if

$$
f(E(\cdot-C)+C)=f, \quad \forall E \in H, \quad x \in \mathbb{R}^{d} .
$$

For trigonometric polynomials we use a bit another definition which is compatible with the above definition of $H$-symmetric functions. We say that $t(\xi)=\sum_{k \in \mathbb{Z}^{d}} h_{k} e^{2 \pi i(k, \xi)}, h_{k} \in \mathbb{C}$, is an $H$ symmetric with respect to the center $c \in \mathbb{R}^{d}$ trigonometric polynomial if

$$
t(\xi)=e^{2 \pi i(c-E c, \xi)} t\left(E^{*} \xi\right), \quad \forall E \in H
$$

and $c-E c \in \mathbb{Z}^{d}, \forall E \in H$. Condition (10) is equivalent to $h_{k}=h_{E(k-c)+c}, \forall k \in \mathbb{Z}^{d}, \forall E \in H$.

Firstly, we show that an $H$-symmetric interpolatory trigonometric polynomial $t$ has some restrictions on the symmetry center. 
Lemma 4 Let $t$ be an interpolatory trigonometric polynomial that is $H$-symmetric with respect to the center $c \in \mathbb{R}^{d}$ and has sum rule of order $n, n \geq 2$. Then $c=E c$ for all $E \in H$.

Proof. From (7) with $\lambda_{\alpha}=\delta_{\mathbf{0} \alpha}, \alpha \in \Delta_{n}$, and the higher chain formula for the linear change of variables it follows that

$$
\left.D^{\beta} t(\xi)\right|_{\xi=\mathbf{0}}=\left.D^{\beta} t\left(E^{*} \xi\right)\right|_{\xi=\mathbf{0}}=(2 \pi i)^{[\beta]} \delta_{\mathbf{0} \beta} \quad \forall \beta \in \Delta_{n} .
$$

Since $t$ is $H$-symmetric, we have that $\forall E \in H$ and $\forall \beta \in \Delta_{n}$

$$
\left.D^{\beta} t\left(M^{*-1} \xi\right)\right|_{\xi=\mathbf{0}}=\left.D^{\beta} e^{2 \pi i\left(c-E c, M^{*-1} \xi\right)} t\left(E^{*} M^{*-1} \xi\right)\right|_{\xi=\mathbf{0}}=(2 \pi i)^{[\beta]}\left(M^{-1}(c-E c)\right)^{\beta} .
$$

Thus, $M^{-1}(c-E c)=\mathbf{0}$, or, equivalently, $c=E c, \forall E \in H$. $\diamond$

The condition $c=E c$ for all $E \in H$ means that either $c=\mathbf{0}$ or $\operatorname{det}\left(I_{d}-E\right)=0$ for all $E \in H$. In the latter case $c$ belongs to the intersection of the null spaces of matrices $I_{d}-E, \forall E \in H$ and condition (10) reduces to $t(\xi)=t\left(E^{*} \xi\right), \forall E \in H$. Thus, we can say that $t$ is $H$-symmetric with respect to any point from the intersection of the null spaces of matrices $I_{d}-E, \forall E \in H$. So, it is no matter how the symmetry center will be chosen. For example, if $H:=\left\{I_{2},\left(\begin{array}{cc}-1 & 0 \\ 0 & 1\end{array}\right)\right\}$, then the symmetry center of trigonometric polynomial defined in Lemma 4 can be at any point on the $y$-axis. For convenience, we assume that the symmetry center for $H$-symmetric interpolatory trigonometric polynomials is $c=\mathbf{0}$. Note that the trigonometric polynomial from Lemma 4 automatically has linear-phase moments (with phase $c=\mathbf{0}$ ) equal to the order of sum rule.

It is known (see, e.g. [8]) that the $H$-symmetry of refinable mask not always carries over to its refinable function. Due to this fact the notion of symmetry group was modified in [8].

A finite set $H_{M}$ of $d \times d$ integer matrices is called a symmetry group with respect to the dilation matrix $M$ if $H_{M}$ is a symmetry group on $\mathbb{Z}^{d}$ such that $M^{-1} E M \in H_{M}, \forall E \in H_{M}$. The last property can be also interpreted as follows: for each $E \in H_{M}$ there exist $E^{\prime} \in H_{M}$ such that

$$
E M=M E^{\prime} \quad \text { or } \quad M^{-1} E=E^{\prime} M^{-1} .
$$

The following statement was shown by B. Han.

Lemma 5 [11, Proposition 2.1] Let $H_{M}$ be a symmetry group with respect to the dilation matrix $M$ and let $m_{0}$ be a refinable mask. Then the mask $m_{0}$ is $H_{M}$-symmetric with respect to the center $c \in \mathbb{R}^{d}$ if and only if the corresponding refinable function $\varphi$ given by (2) is $H_{M}$-symmetric with respect to the center $C \in \mathbb{R}^{d}$, where $C=\left(M-I_{d}\right)^{-1} c$.

Let $M$ be a dilation matrix, $H$ be a symmetry group with respect to the dilation matrix $M$, $c \in \mathbb{R}^{d}$ be an appropriate symmetry center, i.e. $c-E c \in \mathbb{Z}^{d}, \forall E \in H$. It is known that each vector $\beta \in \mathbb{Z}^{d}$ can be uniquely represented as follows: $\beta=M \gamma+s$, where $\gamma \in \mathbb{Z}^{d}$ and $s \in D(M)$. Using this fact it is easy to see that for each digit $s \in D(M)$ and matrix $E \in H$ there exist a unique digit $q \in D(M)$ and a vector $r_{s}^{E} \in \mathbb{Z}^{d}$ such that

$$
E s=M r_{s}^{E}+q+E c-c .
$$

The indices of $r_{s}^{E}$ mean that the vector $r_{s}^{E}$ depends on digit $s$ and matrix $E$.

Denote by $\mathcal{D}:=\left\{\left\langle s_{0}\right\rangle, \ldots,\left\langle s_{m-1}\right\rangle\right\}$ the set of cosets, where the coset is defined by $\left\langle s_{i}\right\rangle:=\{M \beta+$ $\left.s_{i}, \beta \in \mathbb{Z}^{d}\right\}, i=0, \ldots, m-1$. Define the map $\chi$ from the set $H \times \mathcal{D}$ as follows

$$
\chi(E,\langle s\rangle)=E\langle s\rangle:=\left\{E M \beta+E s+c-E c, \beta \in \mathbb{Z}^{d}\right\}, \quad E \in H .
$$

Proposition 6 The set $\mathcal{D}$ is an H-space, where the group action is defined by (14). 
Proof. Let us fix $E \in H$ and show that $E\langle s\rangle \in \mathcal{D}$ for all $s \in D(M)$. Due to (13) there always exists a unique digit $q \in D(M)$ such that $E s+c-E c=M \gamma+q, \gamma \in \mathbb{Z}^{d}$. Therefore

$$
E\langle s\rangle=\left\{E M \beta+E s+c-E c, \beta \in \mathbb{Z}^{d}\right\}=\left\{M\left(E^{\prime} \beta+\gamma\right)+q, \beta \in \mathbb{Z}^{d}\right\}=\langle q\rangle,
$$

where $E^{\prime}$ is from (12). The identity condition in the definition of group action is obviously valid. It remains to show the associativity. Suppose $E, \widetilde{E} \in H$. Then

$$
\widetilde{E} E\langle s\rangle=\left\{\widetilde{E} E M \beta+\widetilde{E} E s+c-\widetilde{E} E c, \beta \in \mathbb{Z}^{d}\right\}=\left\{M \widetilde{E}^{\prime} E^{\prime} \beta+\widetilde{E} E s+c-\widetilde{E} E c, \beta \in \mathbb{Z}^{d}\right\},
$$

where $E^{\prime}, \widetilde{E}^{\prime}$ are from (12). On the other hand, $\widetilde{E}(E\langle s\rangle)=\widetilde{E}\langle q\rangle=\left\{\widetilde{E} M \beta+\widetilde{E} q+c-\widetilde{E} c, \beta \in \mathbb{Z}^{d}\right\}$. Since $q=E s+c-E c-M \gamma$, we see that

$$
\begin{array}{r}
\widetilde{E}(E\langle s\rangle)=\left\{\widetilde{E} M \beta+\widetilde{E}(E s+c-E c-M \gamma)+c-\widetilde{E} c, \beta \in \mathbb{Z}^{d}\right\}= \\
=\left\{M\left(\widetilde{E}^{\prime} \beta-\gamma\right)+\widetilde{E} E s+c-\widetilde{E} E c, \beta \in \mathbb{Z}^{d}\right\} .
\end{array}
$$

Therefore, $\widetilde{E}(E\langle s\rangle)=\widetilde{E} E\langle s\rangle \diamond$.

Thus, Lemmas 2 and 3 are valid for the set $\mathcal{D}$. Hence, the set $\mathcal{D}$ can be split into the disjoint orbits, i.e. there exists a set $\Lambda \subset \mathcal{D}$ such that $\mathcal{D}=\cup_{\langle s\rangle \in \Lambda} H\langle s\rangle$. For convenience, redenote the elements of the set $\Lambda$ by $\left\langle s_{p, 0}\right\rangle$, where $p=0, \ldots, \# \Lambda-1$. As above, $H_{\left\langle s_{p, 0}\right\rangle}$ is the stabilizer of $\left\langle s_{p, 0}\right\rangle$. $\Gamma_{\left\langle s_{p, 0}\right\rangle}$ is a complete set of representatives of the cosets of $H \backslash H_{\left\langle s_{p, 0}\right\rangle}$. The elements of the orbit $H\left\langle s_{p, 0}\right\rangle$ we denote by $\left\langle s_{p, i}\right\rangle$, where $i=0, \ldots, \# \Gamma_{\left\langle s_{p, 0}\right\rangle}-1$. Let us fix index $p$. The matrices of the set $\Gamma_{\left\langle s_{p, 0}\right\rangle}$ we denote by $E^{(i)}$ (or by $E_{p}^{(i)}, E_{t}^{(i)}$ when the matrices from the different sets $\Gamma_{\left\langle s_{p, 0}\right\rangle}$ and $\Gamma_{\left\langle s_{t, 0}\right\rangle}$ are used in a one equality), such that $E^{(i)}\left\langle s_{p, 0}\right\rangle=\left\langle s_{p, i}\right\rangle, E^{(0)}=I_{d}, i=0, \ldots, \# \Gamma_{\left\langle s_{p, 0}\right\rangle}-1$. The digit corresponding to the coset $\left\langle s_{p, i}\right\rangle$ we denote by $s_{p, i}$.

By Lemma 3 for each $\left\langle s_{p, 0}\right\rangle \in \mathcal{D}$ the symmetry group $H$ can be uniquely represented as follows $H=\Gamma_{\left\langle s_{p, 0}\right\rangle} \times H_{\left\langle s_{p, 0}\right\rangle}$, i.e. for each matrix $\widetilde{E}$ in $H$ there exist matrices $E \in \Gamma_{\left\langle s_{p, 0}\right\rangle}$ and $F \in H_{\left\langle s_{p, 0}\right\rangle}$ such that $\widetilde{E}=E F$. The sets $\Gamma_{\left\langle s_{p, 0}\right\rangle}, H_{\left\langle s_{p, 0}\right\rangle}$ can be considered as the "coordinate axes" of the symmetry group $H$. (For each $p, p=0, \ldots, \# \Lambda-1$, these "coordinate axes" of $H$ can be different).

Now we point out some features of the choice of digits and indicate how the matrices from the symmetry group $H$ act on the digits. Let us fix a digit $s_{p, 0} \in D(M)$ and a matrix $E^{(i)} \in \Gamma_{\left\langle s_{p, 0}\right\rangle}$. Since $E^{(i)}\left\langle s_{p, 0}\right\rangle=\left\langle s_{p, i}\right\rangle$, by (13) we get $E^{(i)} s_{p, 0}+c-E^{(i)} c=M r_{p, 0}^{E^{(i)}}+s_{p, i}$, where $r_{p, 0}^{E^{(i)}} \in \mathbb{Z}^{d}$. Let us rechoose the digits $s_{p, i}$ such that $r_{p, 0}^{E^{(i)}}=0$, i.e.

$$
E^{(i)} s_{p, 0}+c-E^{(i)} c=: s_{p, i}, \quad i=0, \ldots, \# \Gamma_{\left\langle s_{p, 0}\right\rangle}-1 .
$$

Throughout the paper we assume that the digits $s_{p, i}$ are chosen in that way.

Suppose $F$ is a matrix from the stabilizer $H_{\left\langle s_{p, 0}\right\rangle}$. Then $F\left\langle s_{p, 0}\right\rangle=\left\langle s_{p, 0}\right\rangle$. Hence by (13)

$$
F s_{p, 0}=M r_{p, 0}^{F}+s_{p, 0}+F c-c,
$$

where $r_{p, 0}^{F} \in \mathbb{Z}^{d}, r_{p, 0}^{F}=M^{-1}\left(c-s_{p, 0}\right)-M^{-1} F\left(c-s_{p, 0}\right)$.

For a matrix $K \in H$ there exist matrices $E^{(i)} \in \Gamma_{\left\langle s_{p, 0}\right\rangle}$ and $F \in H_{\left\langle s_{p, 0}\right\rangle}$ such that $K=E^{(i)} F$. Therefore, $K\left\langle s_{p, 0}\right\rangle=E^{(i)} F\left\langle s_{p, 0}\right\rangle=\left\langle s_{p, i}\right\rangle$. Together with (15) and (16) we obtain that

$$
K s_{p, 0}=E^{(i)} F s_{p, 0}=E^{(i)}\left(M r_{p, 0}^{F}+s_{p, 0}+F c-c\right)=M r_{p, 0}^{K}+s_{p, i}+K c-c,
$$

where $r_{p, 0}^{K}=M^{-1} E^{(i)} M r_{p, 0}^{F}$. Analogously we can represent $K s_{p, i}$. There exist matrices $E^{(j(p, i, K))} \in$ $\Gamma_{\left\langle s_{p, 0}\right\rangle}$ and $F \in H_{\left\langle s_{p, 0}\right\rangle}$ such that $K E^{(i)}=E^{(j(p, i, K))} F$. Therefore, $K\left\langle s_{p, i}\right\rangle=E^{(j(p, i, K))} F\left\langle s_{p, 0}\right\rangle=$ $\left\langle s_{p, j(p, i, K)}\right\rangle$. Here the notation $j(p, \cdot, K)$ means the map from the set of indices $\left\{0, \ldots, \# \Gamma_{\left\langle s_{p, 0}\right\rangle}-1\right\}$ 
to itself and the index $j(p, i, K)$ is uniquely defined by the index $i$ and the matrix $K \in H$ for each $p$. Thus, together with (15) and (16) we obtain

$$
\begin{aligned}
& K s_{p, i}=K E^{(i)} s_{p, 0}+K c-K E^{(i)} c= \\
& E^{(j)} M r_{p, 0}^{F}+s_{p, j}+E^{(j)} F c-c+K c-K E^{(i)} c=M r_{p, i}^{K}+s_{p, j}+K c-c,
\end{aligned}
$$

where $r_{p, i}^{K}=M^{-1} E^{(j)} M r_{p, 0}^{F}$ and $j=j(p, i, K)$.

Let $H$ be a symmetry group on $\mathbb{Z}^{d}$ and $t$ be an $H$-symmetric with respect to the center $c \in \frac{1}{2} \mathbb{Z}^{d}$ trigonometric polynomial. Suppose that $H=H^{i d}:=\left\{I_{d},-I_{d}\right\}$, i.e. the simplest symmetry group. Then condition (10) is equivalent to

$$
t(\xi)=e^{2 \pi i(2 c, \xi)} t(-\xi) \quad \text { or } \quad h_{k}=h_{2 c-k}, \forall k \in \mathbb{Z}^{d} .
$$

Such trigonometric polynomial $t(\xi)$ is called symmetric with respect to the point $c$ (or just pointsymmetric). Easy to see that $H^{i d}$ is a symmetry group with respect to any dilation matrix and Lemma 5 for $H^{i d}$ is always valid. Note that if a symmetry group $H$ contains $H^{i d}$ then for any $H$-symmetric with respect to the point $c$ trigonometric polynomial $t$ we have

$$
D^{e_{\ell}} t(\mathbf{0})=\left.D^{e_{\ell}}\left(e^{2 \pi i(2 c, \xi)} t(-\xi)\right)\right|_{\xi=\mathbf{0}}=2 \pi i 2(c)_{j}-D^{e_{\ell}} t(\mathbf{0})
$$

where for $\ell=1, \ldots, d$, the vectors $e_{\ell} \in \mathbb{Z}^{d}$ are the standard basis of $\mathbb{R}^{d},\left(e_{\ell}\right)_{k}=\delta_{k \ell}, k=1, \ldots, d$. Therefore, $D^{e_{\ell}} t(\mathbf{0})=2 \pi i c^{e \ell}, \ell=1, \ldots, d$, i.e. the phase for linear-phase moments of $t$ must match with the point $c$. The maximal order of linear-phase moments for a point-symmetric trigonometric polynomial must be an even integer (see [16, Proposition 2]).

The following types of symmetry will be considered later. The group

$$
H^{\text {axis }}:=\left\{\operatorname{diag}\left(u_{1}, \ldots, u_{d}\right): u_{j}= \pm 1, j=1, \ldots, d\right\} .
$$

is called the axial symmetry group on $\mathbb{Z}^{d}$. The group

$$
H^{\text {full }}:=\left\{ \pm I_{2}, \pm\left(\begin{array}{cc}
-1 & 0 \\
0 & 1
\end{array}\right), \pm\left(\begin{array}{cc}
0 & 1 \\
-1 & 0
\end{array}\right), \pm\left(\begin{array}{ll}
0 & 1 \\
1 & 0
\end{array}\right)\right\}
$$

is called the 4-fold (or full) symmetry group on $\mathbb{Z}^{2}$.

Example 7 Let us demonstrate the above considerations on the choice of digits. Suppose $H=$ $H^{\text {full }}, M=\left(\begin{array}{ll}2 & 0 \\ 0 & 2\end{array}\right), c=(0,0)$. The set of digits is $D(M)=\{(0,0),(0,1),(1,0),(1,1)\}$. The set of cosets is $\mathcal{D}:=\{\langle(0,0)\rangle,\langle(1,0)\rangle,\langle(0,1)\rangle,\langle(1,1)\rangle\}$. The set of cosets is split into disjoint orbits. There are three orbits since

$$
H\langle(0,0)\rangle=\{\langle(0,0)\rangle\}, H\langle(1,1)\rangle=\{\langle(1,1)\rangle\}, H\langle(1,0)\rangle=\{\langle(1,0)\rangle,\langle(0,1)\rangle\} .
$$

Therefore, $\Lambda=\{\langle(0,0)\rangle,\langle(1,1)\rangle,\langle(1,0)\rangle\}$. Let us renumber the digits according with the above considerations as follows $s_{0,0}=(0,0), s_{1,0}=(1,1), s_{2,0}=(1,0), s_{2,1}=(0,1)$. For each digit $s_{p, 0}$, $p=1,2,3$, the sets $\Gamma_{\left\langle s_{p, 0}\right\rangle}, H_{\left\langle s_{p, 0}\right\rangle}$ can be defined. Namely,

$$
\begin{gathered}
H_{\left\langle s_{0,0}\right\rangle}=H^{\text {full }}, \Gamma_{\left\langle s_{0,0}\right\rangle}=\left\{I_{2}\right\}, H_{\left\langle s_{1,0}\right\rangle}=H^{\text {full }}, \Gamma_{\left\langle s_{1,0}\right\rangle}=\left\{I_{2}\right\} \\
H_{\left\langle s_{2,0}\right\rangle}=\left\{ \pm I_{2}, \pm\left(\begin{array}{cc}
1 & 0 \\
0 & -1
\end{array}\right)\right\}, \Gamma_{\left\langle s_{2,0}\right\rangle}=\left\{I_{2},\left(\begin{array}{cc}
0 & 1 \\
1 & 0
\end{array}\right)\right\} .
\end{gathered}
$$




\section{Preliminary results}

The general scheme for the construction of compactly supported MRA-based dual wavelet systems was developed in [24, 25] (the Mixed Extension Principle). To construct the pair of such wavelet systems one starts with two compactly supported refinable functions $\varphi, \widetilde{\varphi}, \widehat{\varphi}(\mathbf{0})=1, \widehat{\widetilde{\varphi}}(\mathbf{0})=1$, (or its masks $m_{0}, \widetilde{m}_{0}$, respectively, which are trigonometric polynomials). Then one finds trigonometric polynomials $m_{\nu}, \tilde{m}_{\nu}, \nu=1, \ldots, r, r \geq m-1$, called the wavelet masks, such that the following polyphase matrices

$$
\mathcal{M}:=\left(\begin{array}{ccc}
\mu_{00} & \ldots & \mu_{0, m-1} \\
\vdots & \ddots & \vdots \\
\mu_{r, 0} & \ldots & \mu_{r, m-1}
\end{array}\right), \widetilde{\mathcal{M}}:=\left(\begin{array}{ccc}
\widetilde{\mu}_{00} & \ldots & \widetilde{\mu}_{0, m-1} \\
\vdots & \ddots & \vdots \\
\widetilde{\mu}_{r, 0} & \ldots & \widetilde{\mu}_{r, m-1}
\end{array}\right)
$$

satisfy

$$
\mathcal{M}^{*} \widetilde{\mathcal{M}}=I_{m}
$$

Here $\mu_{\nu k}, \widetilde{\mu}_{\nu k} k=0, \ldots, m-1$, are the polyphase components of the wavelet masks $m_{\nu}, \widetilde{m}_{\nu}$ for all $\nu=0, \ldots, r, r \geq m-1$.

The wavelet functions $\psi^{(\nu)}, \widetilde{\psi}^{(\nu)}, \nu=1, \ldots, r, r \geq m-1$, are defined via its Fourier transform

$$
\widehat{\psi^{(\nu)}}(\xi)=m_{\nu}\left(M^{*-1} \xi\right) \widehat{\varphi}\left(M^{*-1} \xi\right), \quad \widehat{\widetilde{\psi}^{(\nu)}}(\xi)=\widetilde{m}_{\nu}\left(M^{*-1} \xi\right) \widehat{\widetilde{\varphi}}\left(M^{*-1} \xi\right) .
$$

If the wavelet functions $\psi^{(\nu)}, \widetilde{\psi}^{(\nu)}, \nu=1, \ldots, r, r \geq m-1$, are constructed as above, then the set of the functions $\left\{\psi_{j k}^{(\nu)}\right\},\left\{\widetilde{\psi}_{j k}^{(\nu)}\right\}$ is said to be a compactly supported MRA-based dual wavelet system generated by the refinable functions $\varphi, \widetilde{\varphi}$ (or their masks $m_{0}, \widetilde{m}_{0}$ ).

For the wavelet functions $\widetilde{\psi}^{(\nu)}, \nu=1, \ldots, r, r \geq m-1$, defined by (20), the $V M^{n}$ property for the corresponding wavelet system is equivalent to the fact that the wavelet masks $\widetilde{m}_{\nu}, \nu=1, \ldots, r$ have vanishing moments up to order $n$.

Suppose that some refinable functions $\varphi, \widetilde{\varphi} \in L_{2}\left(\mathbb{R}^{d}\right)$ generate a compactly supported MRAbased dual wavelet system $\left\{\psi_{j k}^{(\nu)}\right\},\left\{\widetilde{\psi}_{j k}^{(\nu)}\right\}, \nu=1, \ldots, r, r \geq m-1$. A necessary (see [27, Theorem 1]) and sufficient (see [9, Theorems 2.2,2.3]) condition for the wavelet system $\left\{\psi_{j k}^{(\nu)}\right\},\left\{\widetilde{\psi}_{j k}^{(\nu)}\right\}$ to be a pair of dual wavelet frames in $L_{2}\left(\mathbb{R}^{d}\right)$ is that each wavelet system $\left\{\psi_{j k}^{(\nu)}\right\}$ and $\left\{\widetilde{\psi}_{j k}^{(\nu)}\right\}$ has vanishing moments at least of order 1. For instance, in the multidimensional case the explicit method for the construction of compactly supported dual wavelet frames with vanishing moments was suggested in 27. But it is a difficult task to provide various types of symmetry for the wavelet frames.

For the special case of interpolatory refinable masks the scheme can be simplified. Recall that if $m_{0}$ is interpolatory then $\mu_{00} \equiv \frac{1}{\sqrt{m}}$. Moreover, if $m_{0}$ has sum rule of order $n \in \mathbb{N}$ then by Lemma 1 we have that $\lambda_{\beta}=\delta_{\mathbf{0} \beta}$ and conditions (8) are valid. The dual mask can be defined in the following form via its polyphase components

$$
\left(\widetilde{\mu}_{00}, \widetilde{\mu}_{01}, \ldots, \widetilde{\mu}_{0, m-1}\right)=\left(\sqrt{m}\left(1-\sum_{k=1}^{m-1}\left|\mu_{0 k}\right|^{2}\right), \mu_{01}, \ldots, \mu_{0, m-1}\right) .
$$

Not hard to check that with this definition we provide $\sum_{k=0}^{m-1} \mu_{0 k} \overline{\widetilde{\mu}_{0 k}} \equiv 1$. Also, by (8) $\mu_{0 k}(\mathbf{0})=\frac{1}{\sqrt{m}}$, $k=0, \ldots, m-1$. Then $\widetilde{\mu}_{00}(\mathbf{0})=\frac{1}{\sqrt{m}}$ and by Lemma 1 we can conclude that $\widetilde{m}_{0}$ has sum rule at least of order 1 and $\widetilde{\lambda}_{\beta}=\delta_{\mathbf{0} \beta}$. In fact, the mask $\widetilde{m}_{0}$ has sum rule of order $n$ (see [26, Proposition 6]).

The order of sum rule of refinable masks is connected with the order of vanishing moments of wavelet masks. The order of vanishing moments is connected with the approximation order of the corresponding wavelet system (see, e.g. [27]). The method providing the vanishing moments for the wavelet masks is given by 
Lemma 8 ([18, Theorem 10]) Let $\varphi, \widetilde{\varphi} \in S^{\prime}$ be refinable functions with compact support. Let $m_{0}, \widetilde{m}_{0}$ be their refinable masks. Suppose $m_{0}$ is interpolatory and has sum rule of order $n$. Mask $\widetilde{m}_{0}$ is defined by (21). Then there exist a MRA-based dual wavelet system $\left\{\psi_{j k}^{(\nu)}\right\},\left\{\widetilde{\psi}_{j k}^{(\nu)}\right\}, \nu=1, \ldots, m-1$, such that the wavelet system $\left\{\widetilde{\psi}_{j k}^{(\nu)}\right\}$ has the $V M^{n}$ property.

Note that if $\varphi, \widetilde{\varphi}$ are in $L_{2}\left(\mathbb{R}^{d}\right)$, then the MRA-based dual wavelet system in Lemma 8 is a dual wavelet frame. The technique for the extension of the polyphase matrices can be realised as follows. Let us define the part of the row of the polyphase components of $m_{0}$ by $P=\left(\mu_{01}, \ldots, \mu_{0, m-1}\right)$. Then the explicit formulas for the matrix extension are given by

$$
\mathcal{N}=\left(\begin{array}{c|c}
\frac{1}{\sqrt{m}} & P \\
\hline-U P^{*} & \sqrt{m}\left(U-U P^{*} P\right)
\end{array}\right), \quad \widetilde{\mathcal{N}}=\left(\begin{array}{c|c}
\sqrt{m}\left(1-P P^{*}\right) & P \\
\hline-\widetilde{U} P^{*} & \frac{1}{\sqrt{m}} \widetilde{U}
\end{array}\right)
$$

Here $U, \widetilde{U}$ are $(m-1) \times(m-1)$ matrices consisting of trigonometric polynomials such that $U \widetilde{U}^{*} \equiv$ $I_{m-1}$. It follows easily that $\mathcal{N} \widetilde{\mathcal{N}}^{*} \equiv I_{m}$. The matrices $U, \widetilde{U}$, for example, can be taken as follows $U=\widetilde{U}=I_{m-1}$. The next section is devoted to the construction of wavelets using the above technique such that the resulting wavelet systems have some symmetry properties.

\section{Multivariate $H$-symmetric dual wavelet frames}

Firstly, we state two simple lemmas.

Lemma 9 Let $t^{\prime}(\xi)$ be a trigonometric polynomial, $n \in \mathbb{N}, t(\xi):=e^{2 \pi i(a, \xi)} t^{\prime}(\xi)$, where $a \in \mathbb{R}^{d}$. Then $\left.D^{\beta} t^{\prime}(\xi)\right|_{\xi=\mathbf{0}}=(2 \pi i)^{[\beta]} \kappa_{\beta}^{\prime}, \forall \beta \in \Delta_{n}$, if and only if

$$
\left.D^{\beta} t(\xi)\right|_{\xi=\mathbf{0}}=(2 \pi i)^{[\beta]} \sum_{\alpha \in \square_{\beta}} \kappa_{\alpha}^{\prime}\left(\begin{array}{c}
\beta \\
\alpha
\end{array}\right) a^{\beta-\alpha}, \quad \forall \beta \in \Delta_{n} .
$$

Let us fix a symmetry group $H$ on $\mathbb{Z}^{d}$, an appropriate symmetry center $c \in \mathbb{R}^{d}$ and an appropriate matrix dilation $M$ with the set of digits $D(M)$. Now we reformulate the $H$-symmetry condition for a trigonometric polynomial in terms of its polyphase components. The polyphase components of trigonometric polynomial $t$ we enumerate as the corresponding digits: $\tau_{p, i}(\xi), i=0, \ldots, \# \Gamma_{\left\langle s_{p, 0}\right\rangle}-1$, $p=0, \ldots, \# \Lambda-1$.

Lemma $10 A$ trigonometric polynomial $t$ is $H$-symmetric with respect to the appropriate center $c \in \mathbb{R}^{d}$ if and only if for each $p \in\{0, \ldots, \# \Lambda-1\}$ its polyphase components $\tau_{p, i}, i=0, \ldots, \# \Gamma_{\left\langle s_{p, 0}\right\rangle}-1$, satisfy

$$
\tau_{p, i}\left(\left(M^{-1} K M\right)^{*} \xi\right)=e^{2 \pi i\left(r_{p, i}^{K}, \xi\right)} \tau_{p, j}(\xi), \quad \forall K \in H
$$

where index $j=j(p, i, K)$ is defined as above in (18), vector $r_{p, i}^{K}=M^{-1} E^{(j)} M r_{p, 0}^{F}, r_{p, 0}^{F}$ is from (16), the matrices $E^{(j)} \in \Gamma_{\left\langle s_{p, 0}\right\rangle}$ and $F \in H_{\left\langle s_{p, 0}\right\rangle}$ are such that $K E^{(i)}=E^{(j)} F, E^{(i)} \in \Gamma_{\left\langle s_{p, 0}\right\rangle}$.

Proof. Suppose $t$ is an $H$-symmetric with respect to the center $c$ trigonometric polynomial. Then by (3) and (18) we obtain

$$
t\left(K^{*} \xi\right)=\frac{1}{\sqrt{m}} \sum_{p=0}^{\# \Lambda-1} \sum_{i=0}^{\# \Gamma_{\left\langle s_{p, 0}\right\rangle}-1} e^{2 \pi i\left(K s_{p, i}, \xi\right)} \tau_{p, i}\left(M^{*} K^{*} \xi\right)=
$$




$$
\begin{gathered}
\frac{1}{\sqrt{m}} e^{2 \pi i(K c-c, \xi)} \sum_{p=0}^{\# \Lambda-1} \sum_{i=0}^{\# \Gamma_{\left\langle s_{p, 0}\right\rangle}-1} e^{2 \pi i\left(s_{p, j}, \xi\right)} \tau_{p, i}\left(M^{*} K^{*} \xi\right) e^{2 \pi i\left(M r_{p, i}^{K}, \xi\right)}, \\
e^{2 \pi i(K c-c, \xi)} t(\xi)=\frac{1}{\sqrt{m}} e^{2 \pi i(K c-c, \xi)} \sum_{p=0}^{\# \Lambda-1} \sum_{i=0}^{\# \Gamma_{\left\langle s_{p, 0}\right\rangle}-1} e^{2 \pi i\left(s_{p, j}, \xi\right)} \tau_{p, j}\left(M^{*} \xi\right),
\end{gathered}
$$

where $K \in H$, the index $j=j(p, i, K)$ is defined as above, i.e for each $p \in\{0, \ldots, \# \Lambda-1\}$ there exist matrices $E^{(j(p, i, K))} \in \Gamma_{\left\langle s_{p, 0}\right\rangle}$ and $F \in H_{\left\langle s_{p, 0}\right\rangle}$ such that $K E^{(i)}=E^{(j(p, i, K))} F$. Since the polyphase representation is unique with respect to the chosen digits, we get (23). The converse statement is obvious. $\diamond$

According with the above considerations we can rewrite condition (23) in the following form. If $F \in H_{\left\langle s_{p, 0}\right\rangle}$ then

$$
\tau_{p, 0}(\xi)=e^{2 \pi i\left(r_{p, 0}^{F}, \xi\right)} \tau_{p, 0}\left(\left(M^{-1} F M\right)^{*} \xi\right),
$$

where $r_{p, 0}^{F}$ is from (16). Thus, $\tau_{p, 0}(\xi)$ is $M^{-1} H_{\left\langle s_{p, 0}\right\rangle} M$-symmetric with respect to the center $M^{-1}(c-$ $\left.s_{p, 0}\right)$. If $E^{(i)} \in \Gamma_{\left\langle s_{p, 0}\right\rangle}, i=0, \ldots, \# \Gamma_{\left\langle s_{p, 0}\right\rangle}-1$, then

$$
\tau_{p, i}(\xi)=\tau_{p, 0}\left(\left(M^{-1} E^{(i)} M\right)^{*} \xi\right) .
$$

Note that $\tau_{p, i}$ is $M^{-1} H_{\left\langle s_{p, i}\right\rangle} M$-symmetric with respect to the center $M^{-1}\left(c-s_{p, i}\right)$. Indeed, since the group $H_{\left\langle s_{p, i}\right\rangle}$ is conjugate to $H_{\left\langle s_{p, 0}\right\rangle}$, i.e. $H_{\left\langle s_{p, i}\right\rangle}=E^{(i)} H_{\left\langle s_{p, 0}\right\rangle}\left(E^{(i)}\right)^{-1}$, we see that for any $K \in H_{\left\langle s_{p, i}\right\rangle}$ there exists $F \in H_{\left\langle s_{p, 0}\right\rangle}$ such that $K=E^{(i)} F\left(E^{(i)}\right)^{-1}$. Thus, we need to show that $\tau_{p, i}\left(\left(M^{-1} K M\right)^{*} \xi\right) e^{2 \pi i\left(r_{p, i}^{K}, \xi\right)}=\tau_{p, i}(\xi)$, where

$$
r_{p, i}^{K}=M^{-1}\left(c-s_{p, i}\right)-\left(M^{-1} K M\right) M^{-1}\left(c-s_{p, i}\right) .
$$

Indeed, by (24) and (25) we obtain

$$
\begin{gathered}
\tau_{p, i}\left(\left(M^{-1} K M\right)^{*} \xi\right) e^{2 \pi i\left(r_{p, i}^{K}, \xi\right)}=\tau_{p, 0}\left(M^{*} F^{*} E^{(i) *} M^{*-1} \xi\right) e^{2 \pi i\left(r_{p, i}^{K}, \xi\right)}= \\
\tau_{p, 0}\left(M^{*} E^{(i) *} M^{*-1} \xi\right) e^{-2 \pi i\left(r_{p, 0}^{F}, M^{*} E^{(i) *} M^{*-1} \xi\right)} e^{2 \pi i\left(r_{p, i}^{K}, \xi\right)}=\tau_{p, i}(\xi) .
\end{gathered}
$$

The next to last equality is valid due to $r_{p, i}^{K}=M^{-1} E^{(i)} M r_{p, 0}^{F}$.

\subsection{Construction of symmetric refinable masks}

Now we suggest a simple algorithm for the construction of interpolatory refinable masks that are $H$-symmetric with respect to the origin $(c=\mathbf{0})$ and have an arbitrary order of sum rule. Assume that $s_{0,0}=\mathbf{0}$, then $H_{\left\langle s_{0,0}\right\rangle}=H, \Gamma_{\left\langle s_{0,0}\right\rangle}=I_{d}$. Conditions (15), (16), (18) in this case become $E^{(i)} s_{p, 0}=s_{p, i}, F s_{p, 0}=M r_{p, 0}^{F}+s_{p, 0}, K s_{p, i}=M r_{p, i}^{K}+s_{p, j}$, where $j=j(p, i, K)$. Also, since in the interpolatory case $\lambda_{\gamma}=\delta_{\mathbf{0} \gamma}$ then condition (6) is equivalent to condition (8).

Theorem 11 Let $H$ be a symmetry group on $\mathbb{Z}^{d}, M$ be an appropriate matrix dilation, $n \in \mathbb{N}$. Then there exists an interpolatory mask $m_{0}$ that is $H$-symmetric with respect to the origin and has the order of sum rule equal to $n$. This mask $m_{0}$ can be represented by

$$
m_{0}(\xi)=\frac{1}{m}+\frac{1}{\sqrt{m}} \sum_{p=1}^{\# \Lambda-1} \nu_{p}(\xi) .
$$

Here for each $p=1, \ldots, \# \Lambda-1$

$$
\nu_{p}(\xi)=\sum_{i=0}^{\# \Gamma_{\left\langle s_{p, 0}\right\rangle}-1} \frac{e^{2 \pi i\left(s_{p, i}, \xi\right)}}{\# H_{\left\langle s_{p, 0}\right\rangle}} \sum_{F \in H_{\left\langle s_{p}, 0\right\rangle}} G_{0, p, 0}\left(M^{*} F^{*} E^{(i) *} \xi\right) e^{2 \pi i\left(r_{p, 0}^{F}, M^{*} E^{(i) *} \xi\right)},
$$


where $G_{0, p, 0}$ are trigonometric polynomials such that (27) is valid, $E^{(i)} \in \Gamma_{\left\langle s_{p, 0}\right\rangle}$.

Proof. Let us construct the polyphase components $\mu_{0, p, i}(\xi), i=0, \ldots, \# \Gamma_{\left\langle s_{p, 0}\right\rangle}-1, p=$ $0, \ldots, \# \Lambda-1$ such that they satisfy conditions (8) and (23). Therefore, we obtain the required mask $m_{0}$ by (3).

For $p=0$ we can set $\mu_{0,0,0}$ equal to $\frac{1}{\sqrt{m}}$.

Let us fix $p \neq 0$ and construct the polyphase component $\mu_{0, p, 0}(\xi)$. It should satisfy conditions (8) and (24). First of all, define the trigonometric polynomial $G_{0, p, 0}(\xi)$ such that

$$
D^{\beta} G_{0, p, 0}(\mathbf{0})=\frac{(2 \pi i)^{[\beta]}}{\sqrt{m}}\left(-M^{-1} s_{p, 0}\right)^{\beta} \quad \forall \beta \in \Delta_{n} .
$$

Thus, $\mu_{0, p, 0}$ can be obtained as follows

$$
\mu_{0, p, 0}(\xi)=\frac{1}{\# H_{\left\langle s_{p, 0}\right\rangle}} \sum_{F \in H_{\left\langle s_{p}, 0\right\rangle}} G_{0, p, 0}\left(\left(M^{-1} F M\right)^{*} \xi\right) e^{2 \pi i\left(r_{p, 0}^{F}, \xi\right)} .
$$

Let us check that $\mu_{0, p, 0}(\xi)$ is $M^{-1} H_{\left\langle s_{p, 0}\right\rangle} M$-symmetric with respect to the center $-M^{-1} s_{p, 0}$ and conditions (88) are valid. Suppose $\widetilde{F} \in H_{\left\langle s_{p, 0}\right\rangle}$. Then the $M^{-1} H_{\left\langle s_{p, 0}\right\rangle} M$-symmetry of $\mu_{0, p, 0}$ follows from

$$
\begin{gathered}
\mu_{0, p, 0}\left(\left(M^{-1} \widetilde{F} M\right)^{*} \xi\right)=\frac{1}{\# H_{\left\langle s_{p, 0}\right\rangle}} \sum_{F \in H_{\left\langle s_{p}, 0\right\rangle}} G_{0, p, 0}\left(\left(M^{-1} \widetilde{F} F M\right)^{*} \xi\right) e^{2 \pi i\left(M^{-1} \widetilde{F} M r_{p, 0}^{F}, \xi\right)}= \\
\frac{1}{\# H_{\left\langle s_{p, 0}\right\rangle}} \sum_{F \in H_{\left\langle s_{p, 0}\right\rangle}} G_{0, p, 0}\left(\left(M^{-1} \widetilde{F} F M\right)^{*} \xi\right) e^{2 \pi i\left(r_{p, 0}^{\tilde{F} F}-r_{p, 0}^{\widetilde{F}}, \xi\right)}=\mu_{0, p, 0}(\xi) e^{-2 \pi i\left(r_{p, 0}^{\widetilde{F}}, \xi\right)},
\end{gathered}
$$

since $M^{-1} \widetilde{F} M r_{p, 0}^{F}=r_{p, 0}^{\widetilde{F} F}-r_{p, 0}^{\widetilde{F}}$. By Lemma 9, condition (27) is equivalent to

$$
\left.D^{\beta}\left(G_{0, p, 0}(\xi) e^{2 \pi i\left(M^{-1} s_{p, 0}, \xi\right)}\right)\right|_{\xi=\mathbf{0}}=\frac{(2 \pi i)^{[\beta]}}{\sqrt{m}} \delta_{\mathbf{0} \beta} \quad \forall \beta \in \Delta_{n} .
$$

Due to the higher chain rule for the linear change of variables $\xi:=\left(M^{-1} F M\right)^{*} \xi, F \in H_{\left\langle s_{p, 0}\right\rangle}$ we have

$$
\left.D^{\beta}\left(G_{0, p, 0}\left(\left(M^{-1} F M\right)^{*} \xi\right) e^{2 \pi i\left(M^{-1} F s_{p, 0}, \xi\right)}\right)\right|_{\xi=\mathbf{0}}=\frac{(2 \pi i)^{[\beta]}}{\sqrt{m}} \delta_{\mathbf{0} \beta} \quad \forall \beta \in \Delta_{n} .
$$

Then by Lemma 9 and (16) we conclude that $\forall F \in H_{\left\langle s_{p, 0}\right\rangle}$

$$
\left.D^{\beta}\left(G_{0, p, 0}\left(\left(M^{-1} F M\right)^{*} \xi\right) e^{2 \pi i\left(r_{p, 0}^{F}, \xi\right)}\right)\right|_{\xi=\mathbf{0}}=\frac{(2 \pi i)^{[\beta]}}{\sqrt{m}}\left(-M^{-1} s_{p, 0}\right)^{\beta} \quad \forall \beta \in \Delta_{n} .
$$

Hence, condition (8) for $\mu_{0, p, 0}$ is valid.

Next, define $\mu_{0, p, i}(\xi)$ as follows $\mu_{0, p, i}(\xi)=\mu_{0, p, 0}\left(\left(M^{-1} E^{(i)} M\right)^{*} \xi\right)$, where $E^{(i)} \in \Gamma_{\left\langle s_{p, 0}\right\rangle}$. Then the polyphase component $\mu_{0, p, i}(\xi)$ is $M^{-1} H_{\left\langle s_{p, i}\right\rangle} M$-symmetric with respect to the center $-M^{-1} s_{p, i}$.

Show that condition (8) is valid for $\mu_{0, p, i}(\xi)$. We have already proved that

$$
\left.D^{\beta}\left(\mu_{0, p, 0}(\xi) e^{2 \pi i\left(M^{-1} s_{p, 0}, \xi\right)}\right)\right|_{\xi=\mathbf{0}}=\frac{(2 \pi i)^{[\beta]}}{\sqrt{m}} \delta_{\mathbf{0} \beta} \quad \forall \beta \in \Delta_{n}
$$

Hence, by the higher chain rule for the linear change of variables $\xi:=\left(M^{-1} E_{p}^{(i)} M\right)^{*} \xi, E_{p}^{(i)} \in \Gamma_{\left\langle s_{p, 0}\right\rangle}$, we get

$$
\left.D^{\beta}\left(\mu_{0, p, 0}\left(\left(M^{-1} E^{(i)} M\right)^{*} \xi\right) e^{2 \pi i\left(M^{-1} E^{(i)} s_{p, 0}, \xi\right)}\right)\right|_{\xi=\mathbf{0}}=\frac{(2 \pi i)^{[\beta]}}{\sqrt{m}} \delta_{\mathbf{0} \beta} \quad \forall \beta \in \Delta_{n} .
$$


It remains to note that $E^{(i)} s_{p, 0}=s_{p, i}$ and then by Lemma 9

$$
D^{\beta} \mu_{0, p, i}(\mathbf{0})=\frac{(2 \pi i)^{[\beta]}}{\sqrt{m}}\left(-M^{-1} s_{p, i}\right)^{\beta} \quad \forall \beta \in \Delta_{n} .
$$

Hence, we construct the polyphase components $\mu_{0, p, i}(\xi), i=0, \ldots, \# \Gamma_{\left\langle s_{p, 0}\right\rangle}-1, p=0, \ldots, \# \Lambda-1$ such that they satisfy symmetry condition (23) in Lemma 10 and condition (8) with $\lambda_{\gamma}=\delta_{\mathbf{0} \gamma}$. To get the required mask $m_{0}$ it remains to use formula (3) together with (28).

Remark 12 The construction of $H$-symmetric with respect to the point c masks with real coefficients and with sum rule of order $n$ can be done as in Theorem 11 using (26), where $G_{0, p, 0}$ are the same trigonometric polynomial but with real coefficients. Also, the minimal number of non-zero coefficients for masks from Theorem 11 can be achieved if we provide the minimality of non-zero coefficients for $\mu_{0, p, 0}, p=0, \ldots, \# \Lambda-1$.

\subsection{Construction of symmetric wavelet frames}

For the construction of wavelet frames with the symmetry properties we need an $H$-symmetric dual refinable mask $\widetilde{m}_{0}$. It can be defined via its polyphase components by (21). As it was mentioned above $\widetilde{m}_{0}$ has the same order of sum rule as $m_{0}$. If $m_{0}$ is $H$-symmetric then $\widetilde{m}_{0}$ is also $H$-symmetric. Indeed, by (23) it is enough to show that $\widetilde{\mu}_{00}$ is $H$-symmetric. But this is true since $m_{0}$ is $H$ symmetric.

Next, we consider the basic matrix extension as in (22) with $U=\widetilde{U}=I_{m-1}$. Namely,

$$
\mathcal{N}=\left(\begin{array}{c|c}
\frac{1}{\sqrt{m}} & P \\
\hline-P^{*} & \sqrt{m}\left(I_{m-1}-P^{*} P\right)
\end{array}\right), \quad \widetilde{\mathcal{N}}=\left(\begin{array}{c|c}
\sqrt{m}\left(1-P P^{*}\right) & P \\
\hline-P^{*} & \frac{1}{\sqrt{m}} I_{m-1}
\end{array}\right)
$$

Theorem 13 Let $H$ be a symmetry group on $\mathbb{Z}^{d}, M$ be an appropriate matrix dilation, $n \in \mathbb{N}$ and let $m_{0}$ and $\widetilde{m}_{0}$ be $H$-symmetric with respect to the origin masks such that $m_{0}$ has sum rule of order $n, \widetilde{m}_{0}$ is constructed by (21). Then wavelet masks $m_{(p, i)}$ and $\widetilde{m}_{(p, i)}, i=0, \ldots, \# \Gamma_{\left\langle s_{p, 0}\right\rangle}-1$, $p=1, \ldots, \# \Lambda-1$, constructed using the matrix extension (29) have the $V M^{n}$ property and the symmetry properties. Namely, for a fixed number $p=1, \ldots, \# \Lambda-1, m_{(p, i)}$ and $\widetilde{m}_{(p, i)}$ are $H_{\left\langle s_{p, i}\right\rangle}$ symmetric with respect to the center $s_{p, i} ; m_{(p, i)}=m_{(p, 0)}\left(E^{(i) *} \xi\right), \widetilde{m}_{(p, i)}=\widetilde{m}_{(p, 0)}\left(E^{(i) *} \xi\right)$, where $E^{(i)} \in \Gamma_{\left\langle s_{p, 0}\right\rangle}, i=0, \ldots, \# \Gamma_{\left\langle s_{p, 0}\right\rangle}-1$. If $\varphi, \widetilde{\varphi} \in L_{2}\left(\mathbb{R}^{d}\right)$, then the corresponding wavelet system is a dual wavelet frame.

Proof. Let $T_{p}$ denote the row of the polyphase components defined by $T_{p}=\left(\mu_{0, p, 0}, \ldots, \mu_{0, p, N_{p}-1}\right)$, where $N_{p}=\# \Gamma_{\left\langle s_{p, 0}\right\rangle}, p=1, \ldots, \# \Lambda-1$. Let $P=\left(T_{1}, \ldots, T_{\# \Lambda-1}\right)$. Consider the submatrix $I_{m}-P^{*} P$ in (29)

$$
I_{m}-P^{*} P=\left(\begin{array}{cccc}
I_{N_{1}-1}-T_{1}^{*} T_{1} & -T_{1}^{*} T_{2} & \ldots & -T_{1}^{*} T_{\# \Lambda-1} \\
-T_{2}^{*} T_{1} & I_{N_{2}-1}-T_{2}^{*} T_{2} & \ldots & -T_{2}^{*} T_{\# \Lambda-1} \\
\vdots & & \ddots & \vdots \\
-T_{\# \Lambda-1}^{*} T_{1} & \ldots & \ldots & I_{N_{(\# \Lambda-1)}-1}-T_{\# \Lambda-1}^{*} T_{\# \Lambda-1}
\end{array}\right)
$$

The rows of the submatrix are numbered by the double indices $(p, i)$ corresponding to the indices of the polyphase components $\overline{\mu_{0, p, i}}$ in the columns $T_{p}^{*}, p=1, \ldots, \# \Lambda-1$. Consider the row $(p, i)$. The corresponding wavelet mask for this row is defined by $m_{(p, i)}=e^{2 \pi i\left(s_{p, i}, \xi\right)}-\overline{\mu_{0, p, i}\left(M^{*} \xi\right)} m_{0}(\xi)$. The mask $m_{(p, 0)}$ is $H_{\left\langle s_{p, 0}\right\rangle}$-symmetric with respect to the center $s_{p, 0}$. Indeed, due to (16) and (24) for all $F \in H_{\left\langle s_{p, 0}\right\rangle}$ we have 


$$
m_{(p, 0)}\left(F^{*} \xi\right)=e^{2 \pi i\left(F s_{p, 0}, \xi\right)}-\overline{\mu_{0, p, 0}\left(M^{*} F^{*} \xi\right)} m_{0}\left(F^{*} \xi\right)=m_{(p, 0)}(\xi) e^{2 \pi i\left(F s_{p, 0}-s_{p, 0}, \xi\right)} .
$$

Moreover, by (25) we have $m_{(p, i)}(\xi)=m_{(p, 0)}\left(E^{(i) *} \xi\right)$. Similarly, it can be proved that $m_{(p, i)}$ is $H_{\left\langle s_{p, i}\right\rangle}$-symmetric with respect to the center $s_{p, i}$. Namely, for all $\widetilde{F} \in H_{\left\langle s_{p, i}\right\rangle}$

$$
m_{(p, i)}\left(\widetilde{F}^{*} \xi\right)=m_{(p, i)}(\xi) e^{2 \pi i\left(\widetilde{F} s_{p, i}-s_{p, i}, \xi\right)} .
$$

Easy to check that the same symmetric properties are valid for the dual masks, since

$$
\widetilde{m}_{(p, i)}=\frac{e^{2 \pi i\left(s_{p, i}, \xi\right)}}{m}-\frac{\overline{\mu_{0, p, i}\left(M^{*} \xi\right)}}{\sqrt{m}} .
$$

The $V M^{n}$ property for the wavelet masks is provided by Lemma 8

Thus, the matrix extension as in (29) leads to the wavelet masks the are mutually symmetric, i.e. some wavelet masks are reflected or rotated copies of the others. Proposition 2.1 in [11 can be used in order to compute the symmetry centers of wavelet functions. Notice that if $\Gamma_{\left\langle s_{p, 0}\right\rangle}=\left\{I_{d}\right\}$ for all $p=0, \ldots, \# \Lambda-1$, then all wavelet masks constructed by Theorem 13 are $H$-symmetric.

Remark 14 All wavelet functions constructed in Theorem 19 have the $V M^{n}$ property. It is known that the dual wavelet frame provides approximation order $n$ if the dual wavelet functions $\left\{\widetilde{\psi}^{(\nu)}\right\}$ have the $V M^{n}$ property (see Theorem 4 in [27]). So, to obtain dual wavelet frames we just need to require the vanishing moments at least of order 1 for the primal wavelet functions $\left\{\psi^{(\nu)}\right\}$ (as noted above, this is a necessary and sufficient condition for a wavelet system to be a frame). Thus, it is enough to provide the sum rule at least of order 1 for the dual refinable mask $\widetilde{m}_{0}$. The general method for the construction of dual refinable masks with desirable order of sum rule was suggested by B. Han in [7]. Here we slightly modify this method by adding the $H$-symmetry conditions. Let us construct $\widetilde{m}_{0}$ using Theorem 11 with $n=1, \widetilde{\mu}_{0 k}$ be its polyphase components, $k=0, \ldots, m-1$. Now we modify the mask $\widetilde{m}_{0}$ by replacing its first polyphase component $\widetilde{\mu}_{00}=\frac{1}{\sqrt{m}}$ with $\widetilde{\mu}_{00}=\sqrt{m}\left(1-\sum_{k=1}^{m-1} \overline{\mu_{0 k}} \widetilde{\mu}_{0 k}\right)$, where $\mu_{0 k}$ are the polyphase components of the mask $m_{0}$. Easy to see that the modified mask $\widetilde{m}_{0}$ satisfies the following conditions: $\widetilde{m}_{0}$ is $H$-symmetric with respect to the origin, $\widetilde{m}_{0}$ has sum rule at least of order 1 and $\sum_{k=0}^{m-1} \mu_{0 k} \overline{\widetilde{\mu}_{0 k}} \equiv 1$. Analyzing the proof of Theorem 13 we can restate it for the modified dual mask $\widetilde{m}_{0}$. The matrix extension in this case is as follows

$$
\mathcal{N}=\left(\begin{array}{c|c}
\frac{1}{\sqrt{m}} & P \\
\hline-\widetilde{P}^{*} & \sqrt{m}\left(I_{m-1}-\widetilde{P}^{*} P\right)
\end{array}\right), \quad \widetilde{\mathcal{N}}=\left(\begin{array}{c|c}
\sqrt{m}\left(1-\widetilde{P} P^{*}\right) & \widetilde{P} \\
\hline-P^{*} & \frac{1}{\sqrt{m}} I_{m-1}
\end{array}\right),
$$

where $\widetilde{P}$ is the row of the polyphase components of $\widetilde{m}_{0}$ without $\widetilde{\mu}_{00}$. This modification is useful, since the smaller order of sum rule for $\widetilde{m}_{0}$ we demand, the smaller number of non-zero coefficients of $\widetilde{m}_{0}$ we obtain. But also, the smaller order of smoothness we can get for $\widetilde{\varphi}$. And $\widetilde{\varphi}$ may not be in $L_{2}\left(\mathbb{R}^{d}\right)$. So, the desirable order of sum rule for $\widetilde{m}_{0}$ should be appropriately chosen.

\subsection{Symmetrization}

The aim of this subsection is to construct the wavelet masks such that all of them are symmetric in some sense for each matrix from the symmetry group $H$. To do that we extend the definition of $H$-symmetric trigonometric polynomials. Let $t$ be a trigonometric polynomial. Then $t$ has the $H$-symmetry property if for each matrix $E \in H$

$$
t\left(E^{*} \xi\right)=\varepsilon_{E} e^{2 \pi i\left(r_{E}, \xi\right)} t(\xi),
$$

where $\varepsilon_{E} \in \mathbb{C},\left|\varepsilon_{E}\right|=1, r_{E} \in \mathbb{Z}^{d}$. 
Next, we show how to symmetrize the row of the polyphase components of an $H$-symmetric mask, i.e. we want to find a unitary transformation of the row such that each element of the new row has the $H$-symmetry property. This is not always possible. So we restrict our attention to the case when $H$ is an abelian symmetry group on $\mathbb{Z}^{d}$. Then $H$ can be expressed as the direct product of cyclic subgroups due to the fundamental theorem of finite abelian groups. Hence, for any number $p=0, \ldots, \# \Lambda-1$ the set $\Gamma_{<s_{p, 0}>}$ is an abelian group. This is a simple corollary from the fundamental theorem of finite abelian groups since $H_{<s_{p, 0}>}$ is a subgroup of $H$. Thus, group $\Gamma_{<s_{p, 0}>}$ also can be expressed as the direct product of cyclic subgroups. In order to write it down, we need some additional notation. Let us fix $p$ and let $N_{p}=\# \Gamma_{<s_{p, 0}>}$ and $\gamma_{p}$ be the number of cyclic subgroups of $\Gamma_{<s_{p, 0}>}$. Then there exists unique prime numbers $N_{p, i}, i=1, \ldots, \gamma_{p}$ and matrices $\mathcal{E}_{1}, \ldots, \mathcal{E}_{\gamma_{p}} \in \Gamma_{<s_{p, 0}>}$ such that

$$
\Gamma_{<s_{p, 0}>}=\left\{I_{d}, \mathcal{E}_{1}, \ldots, \mathcal{E}_{1}^{N_{p, 1}-1}\right\} \times \cdots \times\left\{I_{d}, \mathcal{E}_{\gamma_{p}}, \ldots, \mathcal{E}_{\gamma_{p}}^{N_{p, \gamma_{p}}-1}\right\} .
$$

Thus, any element $E \in \Gamma_{<s_{p, 0}>}$ can be uniquely represented as follows $E=\prod_{j=1}^{\gamma_{p}} \mathcal{E}_{j}^{k_{j}}$, where $k_{j}$ are some numbers from the sets $\left\{0, \ldots, N_{p, j}-1\right\}$, respectively. Let $L_{p, 1}=1, L_{p, i}=\prod_{j=1}^{i-1} N_{p, j}$, for $i=2, \ldots, \gamma_{p}$ and $L_{p, \gamma_{p}+1}=\prod_{j=1}^{\gamma_{p}} N_{p, j}=N_{p}$. Thus, each number $k \in\left\{0, \ldots, N_{p}-1\right\}$ can be uniquely represented as $k=\sum_{j=1}^{\gamma_{p}} k_{j} L_{p, j}$, where $0 \leq k_{j} \leq N_{p, j}-1$. Or equivalently, there is an one-to-one correspondence between $k$ and $\left(k_{1}, \ldots, k_{\gamma_{p}}\right)$. In other words, we get a mixed radix numeral system for all numbers in the set $\left\{0, \ldots, N_{p}-1\right\}$. Let us renumbered all matrices in the group $\Gamma_{<s_{p, 0}>}$ according with this numeral system. Let $E \in \Gamma_{<s_{p, 0}>}$, then $E=\prod_{j=1}^{\gamma_{p}} \mathcal{E}_{j}^{k_{j}}$, so the matrix $E$ has number $k$, where $k=\sum_{j=1}^{\gamma_{p}} k_{j} L_{p, j}$. We denote the matrix $E$ with number $k$ by $E^{(k)}$.

Let us define addition on the set $\left\{0, \ldots, N_{p}-1\right\}$ as follows: $k \oplus l=\sum_{j=1}^{\gamma_{p}}\left(k_{j} \oplus l_{j}\right) L_{p, j}$, where $k_{j} \oplus l_{j}$ is a summation by module $N_{p, j}$, namely $k_{j} \oplus l_{j}=\left(k_{j}+l_{j}\right) \bmod N_{p, j}$, where $j \in\left\{1, \ldots, \gamma_{p}\right\}$. Not hard to see that, $E^{(k)} E^{(l)}=E^{(k \oplus l)}$, where $E^{(k)}, E^{(l)} \in \Gamma_{<s_{p, 0}>}$.

Using such notations and assumptions we can rewrite some useful properties. Let us fix $p$. Then the matrix $K \in H$ can be represented as $K=E^{(n)} F$, where $E^{(n)} \in \Gamma_{<s_{p, 0}>}, F \in H_{<s_{p, 0}>}$. In this case the map $j(p, i, K)$ simply means $i \oplus n$, since $K E^{(i)}=E^{(i \oplus n)} F$, Thus, (18) can be written as

$$
K s_{p, i}=M r_{p, i}^{K}+s_{p, i \oplus n}+K c-c,
$$

where $r_{p, i}^{K}=M^{-1} E^{(i \oplus n)} M r_{p, 0}^{F}$. Let $m_{0}$ be an $H$-symmetric mask with respect to the origin. Then conditions (23), (25) become

$$
\mu_{0, p, i}\left(\left(M^{-1} E^{(n)} M\right)^{*} \xi\right)=\mu_{0, p, i \oplus n}(\xi), \quad \forall E^{(n)} \in \Gamma_{\left\langle s_{p, 0}\right\rangle},
$$

for $i=0, \ldots, N_{p}-1$ and for matrix $K \in H$ such that $K=E^{(n)} F, E^{(n)} \in \Gamma_{<s_{p, 0}>}, F \in H_{<s_{p, 0}>}$

$$
\mu_{0, p, i}\left(\left(M^{-1} K M\right)^{*} \xi\right)=e^{2 \pi i\left(r_{p, i}^{K}, \xi\right)} \mu_{0, p, i \oplus n}(\xi),
$$

where $r_{p, i}^{K}=M^{-1} E^{(i \oplus n)} M r_{p, 0}^{F}, r_{p, 0}^{F}$ is from (16).

Let $\varepsilon_{N_{p, i}}=e^{\frac{2 \pi i}{N_{p, i}}}$. For any $p \in\{1, \ldots, \# \Lambda-1\}, W_{N_{p, i}}=\frac{1}{\sqrt{N_{p, i}}}\left\{\varepsilon_{N_{p, i}}^{k l}\right\}_{k, l=0, N_{p, i}-1}$ is the matrix of the discrete Fourier transform. It is known that $W_{N_{p, i}}$ is a unitary and symmetric matrix, i.e. $W_{N_{p, i}} W_{N_{p, i}}^{*}=I_{m}, W_{N_{p, i}}^{T}=W_{N_{p, i}}$. Define $\mathcal{W}_{p}=W_{N_{p, 1}} \otimes \cdots \otimes W_{N_{p, \gamma_{p}}}$, where operation $\otimes$ is the Kronecker product. This is also a unitary matrix and the elements can be expressed as follows

$$
\left[\mathcal{W}_{p}\right]_{k, l}=\varepsilon_{N_{p, 1}}^{k_{1} l_{1}} \ldots \varepsilon_{N_{p, \gamma_{p}}}^{k_{\gamma_{p}} l_{\gamma_{p}}},
$$

where $k, l$ are corresponded to $\left(k_{1}, \ldots, k_{\gamma_{p}}\right)$ and $\left(l_{1}, \ldots, l_{\gamma_{p}}\right)$ respectively according to the above mixed radix numeral system. 
Some of the properties of the matrix $\mathcal{W}_{p}$ are

$$
\left[\mathcal{W}_{p}\right]_{k, l}\left[\mathcal{W}_{p}\right]_{n, l}=\left[\mathcal{W}_{p}\right]_{k \oplus n, l}, \quad\left[\mathcal{W}_{p}\right]_{k, l} \overline{\left[\mathcal{W}_{p}\right]_{k, l}}=1, \quad k, l, n=0, \ldots, N_{p, i}-1
$$

The next Lemma shows that $\mathcal{W}_{p}$ symmetrizes the part of the row of polyphase components.

Lemma 15 Let $m_{0}$ be an $H$-symmetric with respect to the origin mask and $\mu_{0, p, i}$ be its polyphase components, $T_{p}$ be the row of polyphase components defined by $T_{p}=\left(\mu_{0, p, 0}, \ldots, \mu_{0, p, N_{p}-1}\right)$. Suppose that $r_{p, 0}^{F}=M^{-1} E M r_{p, 0}^{F}$ for all $F \in H_{<s_{p, 0}>}$ and $E \in \Gamma_{<s_{p, 0}>}$. Then each element of the row $T_{p}^{\prime}:=T_{p} \mathcal{W}_{p}$ has the $H$-symmetry property.

Proof. Denote the elements of the new row $T_{p}^{\prime}$ by $\mu_{0, p, r}^{\prime}, r=0, \ldots, N_{p}-1$. By definition we have $\mu_{0, p, r}^{\prime}(\xi)=\sum_{k=0}^{N_{p}-1}\left[\mathcal{W}_{p}\right]_{k, r} \mu_{0, p, k}(\xi)$. Firstly, we show that $\mu_{0, p, r}^{\prime}(\xi)$ has the $\left(M^{-1} \Gamma_{<s_{p, 0}>} M\right)$-symmetry property. Indeed, by (32) and the properties of $\mathcal{W}_{p}$ we have for all $E^{(n)} \in \Gamma_{<s_{p, 0}>}$

$$
\begin{gathered}
\mu_{0, p, r}^{\prime}\left(\left(M^{-1} E^{(n)} M\right)^{*} \xi\right)=\sum_{k=0}^{N_{p}-1}\left[\mathcal{W}_{p}\right]_{k, r} \mu_{0, p, k}\left(\left(M^{-1} E^{(n)} M\right)^{*} \xi\right)= \\
\sum_{k=0}^{N_{p}-1}\left[\mathcal{W}_{p}\right]_{n \oplus k, r} \overline{\left[\mathcal{W}_{p}\right]_{n, r}} \mu_{0, p, n \oplus k}(\xi)=\overline{\left[\mathcal{W}_{p}\right]_{n, r}} \mu_{0, p, r}^{\prime}(\xi) .
\end{gathered}
$$

Now show that $\mu_{0, p, r}^{\prime}(\xi), r=0, \ldots, N_{p}-1$, are $\left(M^{-1} H_{<s_{p, 0}>} M\right)$-symmetric. Indeed,

$$
\begin{gathered}
\mu_{0, p, r}^{\prime}\left(\left(M^{-1} F M\right)^{*} \xi\right)=\sum_{k=0}^{N_{p}-1}\left[\mathcal{W}_{p}\right]_{k, r} \mu_{0, p, 0}\left(\left(M^{-1} F E^{(k)} M\right)^{*}(\xi)=\right. \\
\sum_{k=0}^{N_{p}-1}\left[\mathcal{W}_{p}\right]_{k, r} \mu_{0, p, 0}\left(\left(M^{-1} E^{(k)} M\right)^{*} \xi\right) e^{-2 \pi i\left(M^{-1} E^{(k)} M r_{p, 0}^{F}, \xi\right)}=\mu_{0, p, r}^{\prime}(\xi) e^{-2 \pi i\left(r_{p, 0}^{F}, \xi\right)} .
\end{gathered}
$$

If $K \in H$ then $K$ can be represented as $K=E^{(n)} F$, where $F \in H_{<s_{p, 0}>}$ and $E^{(n)} \in \Gamma_{<s_{p, 0}>}$. Thus, $\mu_{0, p, r}^{\prime}(\xi)$ has the $H$-symmetry property

$$
\mu_{0, p, r}^{\prime}\left(\left(M^{-1} K M\right)^{*} \xi\right)=\overline{\left[\mathcal{W}_{p}\right]_{n, r}} \mu_{0, p, r}^{\prime}(\xi) e^{-2 \pi i\left(r_{p, 0}^{F}, \xi\right)} \diamond \diamond
$$

According with this Lemma we need a special assumption $r_{p, 0}^{F}=M^{-1} E M r_{p, 0}^{F}$ for all $F \in H_{<s_{p, 0}>}$ and $E \in \Gamma_{<s_{p, 0}>}$ to ensure the $H$-symmetry property for all components of the row $T_{p}^{\prime}$. Notice that this assumption is used only to provide $\left(M^{-1} H_{<s_{p, 0}>} M\right)$-symmetry for $\mu_{0, p, r}^{\prime}(\xi)$.

Define a block diagonal unitary matrix $\mathcal{W}$ as follows: $\mathcal{W}=\operatorname{diag}\left(\mathcal{W}_{1}, \ldots, \mathcal{W}_{\# \Lambda-1}\right)$. Let $m_{0}$ be an $H$-symmetric mask with respect to the origin. Suppose the special assumption is valid for all $p=1, \ldots, \# \Lambda-1$, i.e. $r_{p, 0}^{F}=M^{-1} E M r_{p, 0}^{F}$ for all $F \in H_{<s_{p, 0}>}$ and $E \in \Gamma_{<s_{p, 0}>}$. Then the matrix $\mathcal{W}$ symmetrizes the row $P=\left(T_{1}, \ldots, T_{\# \Lambda-1}\right)$ of the polyphase components of $m_{0}$, namely all elements of the row

$$
P^{s}:=P \mathcal{W}=\left(T_{1} \mathcal{W}_{1}, \ldots, T_{\# \Lambda-1} \mathcal{W}_{\# \Lambda-1}\right)
$$

have the $H$-symmetry property. Wavelets with the $H$-symmetry property can be constructed using matrix $\mathcal{W}$

Theorem 16 Let $H$ be an abelian symmetry group on $\mathbb{Z}^{d}, M$ be an appropriate matrix dilation, $n \in \mathbb{N}$ and let $m_{0}$ and $\widetilde{m}_{0}$ be $H$-symmetric with respect to the origin masks such that $m_{0}$ has sum rule of order $n, \widetilde{m}_{0}$ is constructed by (21). The special assumption is valid for all $p=1, \ldots, \# \Lambda-1$, i.e. $r_{p, 0}^{F}=M^{-1} E M r_{p, 0}^{F}$ for all $F \in H_{<s_{p, 0}>}$ and $E \in \Gamma_{<s_{p, 0}>}$. Then there exist wavelet masks which have the $V M^{n}$ property and the $H$-symmetry property. If $\varphi, \widetilde{\varphi} \in L_{2}\left(\mathbb{R}^{d}\right)$, then the corresponding wavelet system is a dual wavelet frame. 
Proof. Let $T_{p}$ denote the row of the polyphase components defined by $T_{p}=\left(\mu_{0, p, 0}, \ldots, \mu_{0, p, N_{p}-1}\right)$, where $N_{p}=\# \Gamma_{\left\langle s_{p, 0}\right\rangle}, p=1, \ldots, \# \Lambda-1$. Let $P=\left(T_{1}, \ldots, T_{\# \Lambda-1}\right)$. Let us consider the matrix extension (22) with $U=\widetilde{U}=\mathcal{W}^{*}$

$$
\mathcal{N}=\left(\begin{array}{c|c}
\frac{1}{\sqrt{m}} & P \\
\hline-\mathcal{W}^{*} P^{*} & \sqrt{m}\left(\mathcal{W}^{*}-\mathcal{W}^{*} P^{*} P\right)
\end{array}\right), \quad \widetilde{\mathcal{N}}=\left(\begin{array}{c|c}
\sqrt{m}\left(1-P P^{*}\right) & P \\
\hline-\mathcal{W}^{*} P^{*} & \frac{1}{\sqrt{m}} \mathcal{W}^{*}
\end{array}\right)
$$

Let us consider submatrix $\sqrt{m}\left(\mathcal{W}^{*}-\mathcal{W}^{*} P^{*} P\right)$. It can be represented as block matrix

$$
\left.\begin{array}{cccc}
\mathcal{W}^{*}-\mathcal{W}^{*} P^{*} P= & & & \\
\mathcal{W}_{1}^{*}-\left(T_{1} \mathcal{W}_{1}\right)^{*} T_{1} & -\left(T_{1} \mathcal{W}_{1}\right)^{*} T_{2} & \ldots & -\left(T_{1} \mathcal{W}_{1}\right)^{*} T_{\# \Lambda-1} \\
-\left(T_{2} \mathcal{W}_{2}\right)^{*} T_{1} & \mathcal{W}_{2}^{*}-\left(T_{2} \mathcal{W}_{2}\right)^{*} T_{2} & \ldots & -\left(T_{2} \mathcal{W}_{2}\right)^{*} T_{\# \Lambda-1} \\
\vdots & & \ddots & \vdots \\
-\left(T_{\# \Lambda-1} \mathcal{W}_{\# \Lambda-1}\right)^{*} T_{1} & -\left(T_{\# \Lambda-1} \mathcal{W}_{\# \Lambda-1}\right)^{*} T_{2} & \ldots & \mathcal{W}_{\# \Lambda-1}^{*}-\left(T_{\# \Lambda-1} \mathcal{W}_{\# \Lambda-1}\right)^{*} T_{\# \Lambda-1}
\end{array}\right)
$$

Denote the elements of the submatrix $\sqrt{m}\left(\mathcal{W}^{*}-\mathcal{W}^{*} P^{*} P\right)$ as

$$
\sqrt{m}\left(\mathcal{W}^{*}-\mathcal{W}^{*} P^{*} P\right):=\left\{\mu_{(p, i),(t, j)}\right\}_{p=1, \ldots, \# \Lambda \Lambda-1, i=0, \ldots, N_{p}-1}^{t=1, \ldots, \#-1, j=0, \ldots, N_{-}-1}
$$

With this numeration the element $\mu_{(p, i),(t, j)}$ in the submatrix is the element in the block $(p, t)$ with position $(i, j)$. Namely, if $p \neq t$ then

$$
\mu_{(p, i),(t, j)}(\xi)=\sqrt{m}\left[-\left(T_{p} \mathcal{W}_{p}\right)^{*} T_{t}\right]_{i, j}=-\sqrt{m} \overline{\mu_{0, p, i}^{\prime}(\xi)} \mu_{0, t, j}(\xi) ;
$$

if $p=t$ then

$$
\mu_{(p, i),(p, j)}(\xi)=\sqrt{m}\left[\mathcal{W}_{p}^{*}-\left(T_{p} \mathcal{W}_{p}\right)^{*} T_{p}\right]_{i, j}=\sqrt{m}\left(\overline{\left[\mathcal{W}_{p}\right]_{i, j}}-\overline{\mu_{0, p, i}^{\prime}(\xi)} \mu_{0, p, j}(\xi)\right),
$$

where $\mu_{0, p, i}^{\prime}, i=0, \ldots, N_{p}-1$, are the elements of the row $T_{p} \mathcal{W}_{p}, p=1, \ldots, \# \Lambda-1$, . All $\mu_{0, p, i}^{\prime}$ have the $H$-symmetry property by Lemma 15. Now, we check the $H$-symmetry property of $\mu_{(p, i),(t, j)}$. Let us fix $p$ and $t, p \neq t$ and matrix $K \in H$. Then we can represent $K$ as $K=E_{p}^{(k)} F_{p}$, where $E_{p}^{(k)} \in \Gamma_{<s_{p, 0}>}, F_{p} \in H_{<s_{p, 0}>}$, and as $K=E_{t}^{(l)} F_{t}$, where $E_{t}^{(l)} \in \Gamma_{<s_{t, 0}>}, F_{t} \in H_{<s_{t, 0}>}$. Therefore, by (32), (33) and Lemma 15 and we have

$$
\begin{gathered}
\mu_{(p, i),(t, j)}\left(\left(M^{-1} K M\right)^{*} \xi\right)=-\sqrt{m} \overline{\mu_{0, p, i}^{\prime}\left(\left(M^{-1} K M\right)^{*} \xi\right)} \mu_{0, t, j}\left(\left(M^{-1} K M\right)^{*} \xi\right)= \\
-\sqrt{m}\left[\mathcal{W}_{p}\right]_{i, k} e^{2 \pi i\left(r_{p, 0}^{F_{p}}, \xi\right)} \overline{\mu_{0, p, i}^{\prime}(\xi)} e^{-2 \pi i\left(r_{t, 0}^{F_{t}}, \xi\right)} \mu_{0, t, j \oplus l}(\xi)= \\
{\left[\mathcal{W}_{p}\right]_{i, k} e^{2 \pi i\left(r_{p, 0}^{F_{p}}, \xi\right)} e^{-2 \pi i\left(r_{t, 0}^{F_{t}}, \xi\right)} \mu_{(p, i),(t, j \oplus l)}(\xi) .}
\end{gathered}
$$

Let $p=t$ and $K \in H$. Then matrix $K$ can be represented as $K=E_{p}^{(k)} F_{p}$, where $E_{p}^{(k)} \in \Gamma_{<s_{p, 0}>\text {, }}$ $F_{p} \in H_{<s_{p, 0}>}$. Therefore,

$$
\begin{gathered}
\mu_{(p, i),(p, j)}\left(\left(M^{-1} K M\right)^{*} \xi\right)=\sqrt{m}\left(\overline{\left[\mathcal{W}_{p}\right]_{i, j}}-\overline{\mu_{0, p, i}^{\prime}\left(\left(M^{-1} K M\right)^{*} \xi\right)} \mu_{0, p, j}\left(\left(M^{-1} K M\right)^{*} \xi\right)\right)= \\
\sqrt{m}\left(\overline{\left[\mathcal{W}_{p}\right]_{i, j}}-\left[\mathcal{W}_{p}\right]_{i, k} e^{2 \pi i\left(r_{p, 0}^{F_{p}}, \xi\right)} \overline{\mu_{0, p, i}^{\prime}(\xi)} e^{-2 \pi i\left(r_{p, 0}^{F_{p}}, \xi\right)} \mu_{0, p, j \oplus k}(\xi)\right)= \\
\sqrt{m}\left[\mathcal{W}_{p}\right]_{i, k}\left(\overline{\left[\mathcal{W}_{p}\right]_{i, j \oplus k}}-\overline{\mu_{0, p, i}^{\prime}(\xi)} \mu_{0, p, j \oplus k}(\xi)\right)=\left[\mathcal{W}_{p}\right]_{i, k} \mu_{(p, i),(p, j \oplus k)}(\xi) .
\end{gathered}
$$


The elements of the first column $-\mathcal{W}^{*} P^{*}$ in matrix $\mathcal{N}$ we denote by $\mu_{(p, i),(0,0)}$ and they are equal to $\mu_{(p, i),(0,0)}=-\frac{1}{\sqrt{m}} \overline{\mu_{0, p, i}^{\prime}(\xi)}$. Since $\Gamma_{<s_{0,0}>}=\left\{I_{d}\right\}$ and $r_{0,0}^{F}=\mathbf{0}, \forall F \in H_{<s_{0,0}>}$, equation (4.3) for $\mu_{(p, i),(0,0)}$ is as follows

$$
\mu_{(p, i),(0,0)}\left(\left(M^{-1} K M\right)^{*} \xi\right)=\left[\mathcal{W}_{p}\right]_{i, k} e^{2 \pi i\left(r_{p, 0}^{F_{p}}, \xi\right)} \mu_{(p, i),(0,0)}(\xi), \quad \forall K \in H .
$$

Let us fix numbers $p$ and $i$ and construct the wavelet mask $m_{(p, i)}$ from the trigonometric polynomials $\mu_{(p, i),(t, j)}$ by (3). Let us show that the resulting wavelet mask $m_{(p, i)}$ has the $H$-symmetry property. Suppose $K \in H$. Then we can represent matrix $K$ as $K=E_{p}^{(k)} F_{p}$, where $E_{p}^{(k)} \in \Gamma_{<s_{p, 0}>}, F_{p} \in$ $H_{<s_{p, 0}>}$, and $K=E_{t}^{(l)} F_{t}$, where $E_{t}^{(l)} \in \Gamma_{<s_{t, 0}>}, F_{t} \in H_{<s_{t, 0}>}$. Using (31) and (4.3), we get

$$
\begin{gathered}
m_{(p, i)}\left(K^{*} \xi\right)=\frac{1}{\sqrt{m}} \sum_{t=0}^{\# \Lambda-1} \sum_{j=0}^{\# \Gamma_{<s_{t, 0}>-1}^{-1}} e^{2 \pi i\left(K s_{t, j}, \xi\right)} \mu_{(p, i),(t, j)}\left(M^{*} K^{*} \xi\right)= \\
\frac{\left[\mathcal{W}_{p}\right]_{i, k}}{\sqrt{m}} \sum_{t=0}^{\# \Lambda-1} \sum_{j=0}^{\# \Gamma_{<s_{t, 0}>-1}} e^{2 \pi i\left(s_{t, j \oplus l}, \xi\right)} e^{2 \pi i\left(M r_{t, j}^{K}, \xi\right)} \mu_{(p, i),(t, j \oplus l)}\left(M^{*} \xi\right) e^{2 \pi i\left(M r_{p, 0}^{F_{p}}-M r_{t, 0}, \xi\right)}= \\
{\left[\mathcal{W}_{p}\right]_{i, k} e^{2 \pi i\left(M r_{p, 0}^{F_{p}}, \xi\right)} m_{(p, i)}(\xi) .}
\end{gathered}
$$

The last equality is valid since $r_{t, j}^{K}=M^{-1} E_{t}^{(j \oplus l)} M r_{t, 0}^{F_{t}}=r_{t, 0}^{F_{t}}$. Thus, we get the $H$-symmetry property for the wavelet mask $m_{(p, i)}$. In our case the $H$-symmetry property means that when $K=F_{p}$, then

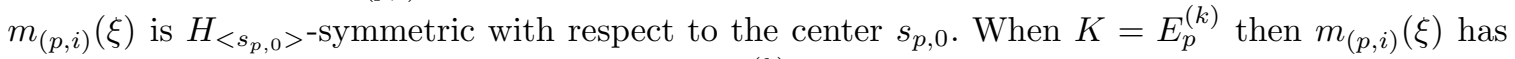
the $\Gamma_{<s_{p, 0}>}$-symmetry property, namely, $m_{(p, i)}\left(E_{p}^{(k) *} \xi\right)=\left[\mathcal{W}_{p}\right]_{i, k} m_{(p, i)}(\xi)$. Not hard to see that the dual wavelet masks $\widetilde{m}_{(p, i)}$ have the same $H$-symmetry property as $m_{(p, i)}$.

The $V M^{n}$ property for the wavelet masks is provided by Lemma 8 , $\diamond$

Note that the comments in Remark 14 are also true for Theorem [16.

\section{$5 \quad$ Examples}

In this section we give several examples to illustrate the main results of this paper. All examples are based on the construction of $H$-symmetric interpolatory refinable masks $m_{0}$ and $\widetilde{m}_{0}$ and wavelet masks $m_{\nu}, \widetilde{m}_{\nu}, \nu=1, \ldots, m$ by Theorems 11, 13 and 16.

1. Let $H$ be a hexagonal symmetry group, namely,

$$
H=\left\{ \pm I_{2}, \pm\left(\begin{array}{ll}
0 & 1 \\
1 & 0
\end{array}\right), \pm\left(\begin{array}{cc}
1 & 0 \\
1 & -1
\end{array}\right), \pm\left(\begin{array}{cc}
1 & -1 \\
1 & 0
\end{array}\right), \pm\left(\begin{array}{cc}
0 & 1 \\
-1 & 1
\end{array}\right), \pm\left(\begin{array}{cc}
-1 & 1 \\
0 & 1
\end{array}\right)\right\}
$$

$c=\mathbf{0}, M=\left(\begin{array}{cc}2 & -1 \\ 1 & 1\end{array}\right)$. The set of digits is $D(M)=\left\{s_{0}=(0,0), s_{1}=(0,1), s_{2}=(0,-1)\right\}, m=3$.

Let us construct a refinable mask that is $H$-symmetric with respect to the origin and has sum rule of order $n=3$. In our case, the digits are renumbered as $s_{0,0}=(0,0), s_{1,0}=(0,1), s_{1,1}=(0,-1)$. And $H_{<s_{0,0}>}=H, \Gamma_{<s_{0,0}>}=\left\{I_{2}\right\}$,

$$
H_{<s_{1,0}>}=\left\{I_{2},\left(\begin{array}{cc}
0 & 1 \\
1 & 0
\end{array}\right),\left(\begin{array}{cc}
-1 & 0 \\
-1 & 1
\end{array}\right),\left(\begin{array}{ll}
-1 & 1 \\
-1 & 0
\end{array}\right),\left(\begin{array}{cc}
0 & -1 \\
1 & -1
\end{array}\right),\left(\begin{array}{cc}
1 & -1 \\
0 & -1
\end{array}\right)\right\},
$$

$\Gamma_{<s_{0,0}>}=\left\{I_{2},\left(\begin{array}{cc}1 & 0 \\ 1 & -1\end{array}\right)\right\}$. According to Theorem [1], the mask $m_{0}$ can be constructed as follows 


$$
m_{0}:\left(\begin{array}{ccccc}
0 & 0 & -\frac{1}{27} & 0 & -\frac{1}{27} \\
0 & 0 & \frac{4}{27} & \frac{4}{27} & 0 \\
-\frac{1}{27} & \frac{4}{27} & \frac{1}{3} & \frac{4}{27} & -\frac{1}{27} \\
0 & \frac{4}{27} & \frac{4}{27} & 0 & 0 \\
-\frac{1}{27} & 0 & -\frac{1}{27} & 0 & 0
\end{array}\right)
$$

with support in $[-2,2]^{2} \cap \mathbb{Z}^{2}$. Note that $\mu_{00}=\frac{1}{\sqrt{3}}$. The corresponding refinable function $\varphi$ is in $L_{2}\left(\mathbb{R}^{2}\right)$ and $\nu_{2}(\varphi) \geq 1.8959$. The Sobolev smothness exponent is calculated by [10, Theorem 7.1]. The dual mask $\widetilde{m}_{0}$ is

$$
\widetilde{m}_{0}:\left(\begin{array}{ccccccccc}
0 & 0 & 0 & 0 & 0 & 0 & -\frac{2}{243} & 0 & 0 \\
0 & 0 & 0 & 0 & \frac{8}{243} & 0 & 0 & \frac{8}{243} & 0 \\
0 & 0 & -\frac{2}{243} & 0 & -\frac{1}{27} & -\frac{16}{243} & -\frac{1}{27} & 0 & -\frac{2}{243} \\
0 & 0 & 0 & -\frac{16}{243} & \frac{4}{27} & \frac{4}{27} & -\frac{16}{243} & 0 & 0 \\
0 & \frac{8}{243} & -\frac{1}{27} & \frac{4}{27} & \frac{47}{81} & \frac{4}{27} & -\frac{1}{27} & \frac{8}{243} & 0 \\
0 & 0 & -\frac{16}{243} & \frac{4}{27} & \frac{4}{27} & -\frac{16}{243} & 0 & 0 & 0 \\
-\frac{2}{243} & 0 & -\frac{1}{27} & -\frac{16}{243} & -\frac{1}{27} & 0 & -\frac{2}{243} & 0 & 0 \\
0 & \frac{8}{243} & 0 & 0 & \frac{8}{243} & 0 & 0 & 0 & 0 \\
0 & 0 & -\frac{2}{243} & 0 & 0 & 0 & 0 & 0 & 0
\end{array}\right)
$$

with support in $[-4,4]^{2} \cap \mathbb{Z}^{2}$. The corresponding refinable function $\widetilde{\varphi}$ is in $L_{2}\left(\mathbb{R}^{2}\right)$ and $\nu_{2}(\widetilde{\varphi}) \geq$ 0.7852 . Since the hexagonal symmetry group $H$ is not abelian, the wavelet masks are constructed by Theorem 13 ;

$$
\begin{aligned}
& m_{1}:\left(\begin{array}{ccccccccc}
0 & 0 & 0 & 0 & -\frac{1}{243} & 0 & -\frac{1}{243} & 0 & 0 \\
0 & 0 & 0 & 0 & \frac{4}{243} & \frac{8}{243} & 0 & \frac{4}{243} & 0 \\
0 & 0 & -\frac{1}{243} & \frac{8}{243} & \frac{1}{27} & -\frac{8}{243} & -\frac{2}{27} & 0 & -\frac{1}{243} \\
0 & 0 & 0 & -\frac{8}{243} & -\frac{8}{81} & -\frac{4}{27} & -\frac{8}{243} & \frac{8}{243} & 0 \\
0 & \frac{4}{243} & -\frac{2}{27} & -\frac{4}{27} & \frac{64}{81} & -\frac{8}{81} & \frac{1}{27} & \frac{4}{243} & -\frac{1}{243} \\
0 & 0 & -\frac{8}{243} & -\frac{8}{81} & -\frac{4}{27} & -\frac{8}{243} & \frac{8}{243} & 0 & 0 \\
-\frac{1}{243} & \frac{8}{243} & \frac{1}{27} & -\frac{8}{243} & -\frac{2}{27} & 0 & -\frac{1}{243} & 0 & 0 \\
0 & \frac{4}{243} & \frac{8}{243} & 0 & \frac{4}{243} & 0 & 0 & 0 & 0 \\
-\frac{1}{243} & 0 & -\frac{1}{243} & 0 & 0 & 0 & 0 & 0 & 0
\end{array}\right), \\
& \tilde{m}_{1}:\left(\begin{array}{ccccc}
0 & 0 & \frac{1}{27} & 0 & 0 \\
0 & 0 & 0 & -\frac{4}{27} & 0 \\
0 & -\frac{4}{27} & \frac{1}{3} & 0 & \frac{1}{27} \\
0 & 0 & -\frac{4}{27} & 0 & 0 \\
\frac{1}{27} & 0 & 0 & 0 & 0
\end{array}\right), m_{2}=m_{1}\left(E^{*} \xi\right), \widetilde{m}_{2}=\widetilde{m}_{1}\left(E^{*} \xi\right),
\end{aligned}
$$

where $E=\left(\begin{array}{cc}1 & 0 \\ 1 & -1\end{array}\right)$. The bold element in the matrices corresponds to the coefficient $h_{\mathbf{0}}$ of the masks. The wavelet masks $m_{1}, m_{2}, \widetilde{m}_{1}, \widetilde{m}_{2}$ have vanishing moments of order 3 , the masks $m_{1}, \widetilde{m}_{1}$ are $H_{<s_{1,0}>}$-symmetric.

2. Let $H=H^{a x i s}, M=\left(\begin{array}{ll}3 & 0 \\ 0 & 2\end{array}\right)$. Let us construct a refinable mask $m_{0}$ that is $H$-symmetric with respect to the origin and has sum rule of order $n=1$. In our case, the digits are renumbered as $s_{0,0}=(0,0), s_{1,0}=(0,1), s_{2,0}=(1,0), s_{2,1}=(-1,0), s_{3,0}=(1,1), s_{3,1}=(-1,1), H_{\left\langle s_{0,0}\right\rangle}=$ $H_{<s_{1,0}>}=H, \Gamma_{<s_{0,0}>}=\Gamma_{<s_{1,0}>}=\left\{I_{2}\right\}$,

$$
H_{<s_{2,0}>}=H_{<s_{3,0}>}=\left\{I_{2},\left(\begin{array}{cc}
1 & 0 \\
0 & -1
\end{array}\right)\right\}, \quad \Gamma_{<s_{2,0}>}=\Gamma_{<s_{3,0}>}=\left\{I_{2},\left(\begin{array}{cc}
-1 & 0 \\
0 & 1
\end{array}\right)\right\} .
$$


The refinable mask $m_{0}$ constructed by Theorem 11 and the dual mask $\widetilde{m}_{0}$ constructed by (21) are

$$
m_{0}:\left(\begin{array}{ccc}
\frac{1}{12} & \frac{1}{12} & \frac{1}{12} \\
\frac{1}{6} & \frac{1}{6} & \frac{1}{6} \\
\frac{1}{12} & \frac{1}{12} & \frac{1}{12}
\end{array}\right), \quad \widetilde{m}_{0}:\left(\begin{array}{ccc}
0 & -\frac{1}{8} & 0 \\
\frac{1}{12} & \frac{1}{12} & \frac{1}{12} \\
\frac{1}{6} & \frac{5}{12} & \frac{1}{6} \\
\frac{1}{12} & \frac{1}{12} & \frac{1}{12} \\
0 & -\frac{1}{8} & 0
\end{array}\right)
$$

with support in $[-1,1]^{2} \cap \mathbb{Z}^{2}$ and in $[-1,1] \times[-2,2] \cap \mathbb{Z}^{2}$ respectively. Since the axial symmetry group is an abelian group, the wavelet masks can be constructed by Theorem 16. Note that the special assumption is valid in this case. To avoid roots in the coefficients of wavelet mask we take matrix $\mathcal{W}$ as

$$
\mathcal{W}=\left(\begin{array}{ccccc}
1 & 0 & 0 & 0 & 0 \\
0 & 1 & 1 & 0 & 0 \\
0 & 1 & -1 & 0 & 0 \\
0 & 0 & 0 & 1 & 1 \\
0 & 0 & 0 & 1 & -1
\end{array}\right), \quad \widetilde{\mathcal{W}}=\left(\begin{array}{ccccc}
1 & 0 & 0 & 0 & 0 \\
0 & \frac{1}{2} & \frac{1}{2} & 0 & 0 \\
0 & \frac{1}{2} & -\frac{1}{2} & 0 & 0 \\
0 & 0 & 0 & \frac{1}{2} & \frac{1}{2} \\
0 & 0 & 0 & \frac{1}{2} & -\frac{1}{2}
\end{array}\right)
$$

where $\widetilde{\mathcal{W}}$ is a paraunitary matrix for $\mathcal{W}$, i.e. $\mathcal{W}^{*} \widetilde{\mathcal{W}}=I_{5}$. The wavelet masks $m_{1}, m_{2}, m_{3}, m_{4}, m_{5}$ are

$$
\begin{gathered}
\left(\begin{array}{ccc}
-\frac{1}{24} & -\frac{1}{24} & -\frac{1}{24} \\
-\frac{1}{12} & -\frac{1}{12} & -\frac{1}{12} \\
-\frac{1}{12} & \frac{11}{12} & -\frac{1}{12} \\
-\frac{1}{12} & -\frac{1}{12} & -\frac{1}{12} \\
-\frac{1}{24} & -\frac{1}{24} & -\frac{1}{24}
\end{array}\right),\left(\begin{array}{ccc}
-\frac{1}{6} & -\frac{1}{6} & -\frac{1}{6} \\
\frac{2}{3} & -\frac{1}{3} & \frac{2}{3} \\
-\frac{1}{6} & -\frac{1}{6} & -\frac{1}{6}
\end{array}\right),\left(\begin{array}{ccc}
0 & 0 & 0 \\
-1 & 0 & 1
\end{array}\right), \\
\left(\begin{array}{ccc}
-\frac{1}{12} & -\frac{1}{12} & -\frac{1}{12} \\
-\frac{1}{6} & -\frac{1}{6} & -\frac{1}{6} \\
\frac{5}{6} & -\frac{1}{6} & \frac{5}{6} \\
-\frac{1}{6} & -\frac{1}{6} & -\frac{1}{6} \\
-\frac{1}{12} & -\frac{1}{12} & -\frac{1}{12}
\end{array}\right),\left(\begin{array}{ccc}
-1 & 0 & 1 \\
0 & 0 & 0
\end{array}\right),
\end{gathered}
$$

The dual wavelet masks $\widetilde{m}_{1}, \widetilde{m}_{2}, \widetilde{m}_{3}, \widetilde{m}_{4}, \widetilde{m}_{5}$ are

$$
\begin{gathered}
\left(\begin{array}{ccc}
0 & -\frac{1}{12} & 0 \\
0 & \frac{1}{6} & 0 \\
0 & -\frac{1}{12} & 0
\end{array}\right),\left(\begin{array}{ccc}
0 & 0 & 0 \\
\frac{1}{12} & -\frac{1}{6} & \frac{1}{12}
\end{array}\right),\left(\begin{array}{ccc}
0 & 0 & 0 \\
-\frac{1}{12} & 0 & \frac{1}{12}
\end{array}\right), \\
\left(\begin{array}{ccc}
0 & -\frac{1}{12} & 0 \\
\frac{1}{12} & 0 & \frac{1}{12} \\
0 & -\frac{1}{12} & 0
\end{array}\right),\left(\begin{array}{ccc}
-\frac{1}{12} & 0 & \frac{1}{12} \\
0 & 0 & 0
\end{array}\right),
\end{gathered}
$$

where the bold element in the matrices corresponds to the coefficient $h_{\mathbf{0}}$ of the masks. All wavelet masks have vanishing moments of order 1 and the $H^{\text {axis }}$-symmetry property.

\section{References}

[1] G. Andaloro, M. Cotronei, L. Puccio, A new class of non-separable symmetric wavelets for image processing, Communications to simai congress, 3 (2009) 324-336.

[2] P. J. Cameron, Permutation Groups, Cambridge University Press, 1999.

[3] O. Christensen, Frames and bases: An introductory course, Birkhauser, 2008.

[4] Dyn N., Skopina M., Decompositions of trigonometric polynomials related to multivariate subdivision schemes, Adv. in Comp. Math. 38, No. 2 (2013) 321-349. 
[5] M. Ehler, B. Han, Wavelet bi-frames with few generators from multivariate refinable functions, Appl. Comput. Harmon. Anal., vol. 25 (2008), no. 3, 407-414.

[6] B. Han, Analysis and construction of optimal multivariate biorthogonal wavelets with compact support, SIAM J. on Mathematical Anal., Vol. 31, No.2 (1999/2000), 274-304

[7] B. Han, Construction of multivariate biorthogonal wavelets by CBC algorithm, Wavelet analysis and multiresolution methods (Urbana-Champaign, IL, 1999), 105-143, Lecture Notes in Pure and Appl. Math., 212, Dekker, New York, 2000.

[8] B. Han, Symmetry property and construction of wavelets with a general dilation matrix, Linear Algebra Appl. 353 (2002) 207-225.

[9] B. Han, Compactly supported tight wavelet frames and orthonormal wavelets of exponential decay with a general dilation matrix, J. Comput. Appl. Math. 155 (2003) 43-67.

[10] B. Han, Computing the smoothness exponent of a symmetric multivariate refinable function, SIAM J. on Matrix Anal. and its Appl., Vol. 24 (2003), No. 3, 693-714.

[11] B. Han, Symmetric multivariate orthogonal refinable functions, Appl. Comput. Harmon. Anal. 17 (2004) 277-292.

[12] B. Han, Matrix extension with symmetry and applications to symmetric orthonormal complex M-wavelets, J. Fourier Anal. Appl. 15 (2009) 684-705.

[13] Bin Han and Zuowei Shen, Dual wavelet frames and Riesz bases in Sobolev spaces, Constructive Approximation, Vol. 29 (2009), Issue 3, 369-406.

[14] B. Han, Symmetric orthonormal complex wavelets with masks of arbitrarily high linear-phase moments and sum rules, Adv. Comput. Math. 32 (2010) 209-237.

[15] B. Han, X. S. Zhuang, Matrix extension with symmetry and its application to symmetric orthonormal multiwavelets, SIAM J. Math. Anal. 42 (2010) 2297-2317.

[16] B. Han, Symmetric orthogonal filters and wavelets with linear-phase moments, J. Comput. Appl. Math. 236 (2011) 482-503.

[17] Q. T. Jiang, D.K. Pounds, Highly symmetric bi-frames for triangle surface multiresolution processing, Appl. Comput. Harmon. Anal. 31 (2011) 370-391.

[18] S. Karakazyan, M. Skopina, M. Tchobanou, Symmetric multivariate wavelets, Int. J. Wavelets Multiresolut. Inf. Process. 7 (2009) 313-340.

[19] K. Koch, Multivariate Symmetric Interpolating Scaling Vectors with Duals, J. of Fourier Anal. and Apps. 15 (2009) 1-30.

[20] A. Krivoshein, On construction of multivariate symmetric MRA-based wavelets, Appl. Comput. Harmon. Anal. 36 (2014) 215-238.

[21] A. Krivoshein, M. Skopina, Approximation by frame-like wavelet systems, Applied and Computational Harmonic Analysis, Volume 31, Issue 3, November 2011, Pages 410-428.

[22] I. Ya. Novikov, V. Yu. Protasov and M. A. Skopina, Wavelet Theory, Amer. Math. Soc, 2011.

[23] A. Petukhov, Construction of symmetric orthogonal bases of wavelets and tight wavelet frames with integer dilation factor. Appl. Comput. Harmon. Anal. 17 (2004) 198-210. 
[24] A. Ron, Z. Shen, Affine systems in $L_{2}\left(R^{d}\right)$ : the analysis of the analysis operator, J. Func. Anal. 148 (1997) 408-447.

[25] A. Ron, Z. Shen, Affine systems in $L_{2}\left(R^{d}\right)$ II: dual systems. J. Fourier Anal. Appl. 3 (1997) 617-637

[26] M. Skopina, On construction of multivariate wavelets with vanishing moments, Appl. Comput. Harmon. Anal. 20 (2006) 375-390.

[27] M. Skopina, On construction of multivariate wavelet frames, Appl. Comput. Harmon. Anal. 27 (2009) 55-72.

[28] X.S. Zhuang, Matrix extension with symmetry and construction of biorthogonal multiwavelets with any integer dilation, Appl. Comput. Harmon. Anal. 33 (2012) 159-181. 